\title{
The LMTK1-TBC1D9B-Rab11A Cascade Regulates Dendritic Spine Formation via Endosome Trafficking
}

\author{
DHironori Nishino, ${ }^{1}$ Taro Saito, ${ }^{1}$ Ran Wei, ${ }^{1}$ Tetsuya Takano, ${ }^{1}$ Koji Tsutsumi, ${ }^{1}$ Makoto Taniguchi, ${ }^{2}$ Kanae Ando, ${ }^{1}$ \\ Mineko Tomomura, ${ }^{3}{ }^{-}$Mitsunori Fukuda, ${ }^{4}$ and ${ }^{\circledR}$ Shin-ichi Hisanaga ${ }^{1}$ \\ ${ }^{1}$ Department of Biological Sciences, Tokyo Metropolitan University, Minami-osawa, Hachioji, Tokyo 192-0397, Japan, ${ }^{2}$ Department of Neuroscience, \\ Medical University of South Carolina, Charleston, South Carolina 29425, ${ }^{3}$ Department of Oral Health Sciences, Meikai University School of Dentistry, \\ Urayasu, Chiba 279-9950, Japan, and ${ }^{4}$ Department of Integrative Life Sciences, Graduate School of Life Sciences, Tohoku University, Aobayama, Aoba-ku, \\ Sendai, Miyagi 980-8578, Japan
}

Dendritic spines are postsynaptic protrusions at excitatory synapses that are critical for proper neuronal synaptic transmission. While lipid and protein membrane components are necessary for spine formation, it is largely unknown how they are recruited to developing spines. Endosomal trafficking is one mechanism that may influence this development. We recently reported that Lemur kinase $1 \mathrm{~A}$ (LMTK1A), a membrane-bound Ser/Thr kinase, regulates trafficking of endosomes in neurons. LMTK1 has been shown to be a p35 Cdk5 activator-binding protein and a substrate for Cdk5-p35; however, its neuronal function has not been sufficiently studied. Here, we investigate the role of LMTK1 in spine formation. Depletion of LMTK1 increases spine formation, maturation, and density in primary cultured neurons and in mouse brain of either sex. Additionally, expression of kinase-negative LMTK1 stimulates spine formation in primary neurons and in vivo. LMTK1 controls spine formation through Rab11, a regulator of recycling endosome trafficking. We identify TBC1D9B, a Rab11A GTPase-activating protein (Rab11A GAP), as a LMTK1 binding protein, and find that TBC1D9B mediates LMTK1 activity on Rab11A. TBC1D9B inactivates Rab11A under the control of LMTK1A. Further, by analyzing the effect of decreased TBC1D9B expression in primary neurons, we demonstrate that TBC1D9B indeed regulates spine formation. This is the first demonstration of the biological function of TBC1D9B. Together, with the regulation of LMTK1 by Cdk5-p35, we propose the Cdk5-LMTK1-TBC1D9B-Rab11A cascade as a novel signaling mechanism regulating endosomal transport for synapse formation and function.

Key words: Cdk5; endosome; GAP; membrane trafficking; Rab; spine

Significance Statement

Dendritic spines are postsynaptic specializations essential for synaptic transmission. However, it is not known how critical membrane components are recruited to spines for their formation. Endosomal trafficking is one such mechanism that may mediate this process. Here we investigate regulators of endosomal trafficking and their contribution to spine formation. We identify two novel factors, LMTK1 and TBC1D9B, which regulate spine formation upstream of Rab11A, a small GTPase. LMTK1 is a membrane bound Ser/Thr kinase regulated by Cdk5-p35, and TBC1D9B is a recently identified Rab11 GAP. LMTK1 controls the GAP activity of TBC1D9B on Rab11A, and TBC1D9B mediates the LMTK1 activity on Rab11A. We propose the Cdk5-LMTK1TBC1D9B-Rab11A cascade as a novel mechanism controlling spine formation and function.

\section{Introduction}

The synapse is a specialized structure for transmitting excitatory or inhibitory signals from neuron to neuron (Choquet and

\footnotetext{
Received Dec. 20, 2018; revised 0ct. 4, 2019; accepted 0ct. 10, 2019.

Author contributions: H.N. and S.-i.H. designed research; H.N., T.S., and R.W. performed research; H.N., T.S., R.W., T.T., K.T., M. Taniguchi, K.A., M.F., and S.-i.H. analyzed data; H.N. wrote the first draft of the paper; H.N., T.T., and S.-i.H. edited the paper; T.S., T.T., K.T., M. Tomomura, and M.F. contributed unpublished reagents/analytic tools; S.-i.H. wrote the paper.

This work was supported in part by Ministry of Education, Culture, Sports, Science, and Technology, Scientific Research on Innovation Area (Brain Protein Aging and Dementia Control) of Japan Grant-in-Aid Project 26117004 to S.-i.H. and Ministry of Education, Culture, Sports, Science, and Technology Grant-in-Aid $16 K 07060$ to S.-i.H. We
}

Triller, 2013). Synapses are composed of presynaptic and postsynaptic terminals. In particular, a postsynaptic region of excit-

\footnotetext{
thank Dr. Jamie Courtland (Department of Cell Biology, Duke University Medical School) for reading the manuscript; Dr. Ko Sato (Department of Chemistry, Tokyo Metropolitan University) for discussion; and Dr. Miyuki Takahashi for statistical analysis.

The authors declare no competing financial interests.

Correspondence should be addressed to Shin-ichi Hisanaga at hisanaga-shinichi@tmu.ac.jp.

T. Takano's present address: Department of Cell Biology, Duke University Medical School, Durham, NC 27703.

K. Tsutsumi's present address: Division of Cell Biology, Department of Biosciences, School of Science, Kitasato University, Kanagawa 252-0373, Japan.

https://doi.org/10.1523/JNEUROSCI.3209-18.2019

Copyright $\odot 2019$ the authors
} 
atory synapses forms a protrusion called a dendritic spine (Calabrese et al., 2006). Upon LTP, spines increase in size and receptor density; whereas after LTD, spines shrink and display a reduced number of receptors (Bosch and Hayashi, 2012). The number and size of spines are carefully regulated depending on synaptic activity. Indeed, hyperformation and hypoformation of synapses are reported in patients with psychiatric disorders and neurodegenerative diseases, respectively (Hutsler and Zhang, 2010; Spires-Jones and Hyman, 2014). Therefore, maintenance of synaptic structure and function is crucial to proper brain functioning. While numerous research studies have attempted to reveal the mechanism of synapse formation, it is not fully understood how the number and size of spines are determined.

The process of spine formation is well understood from a morphological standpoint; filopodial protrusions form first, and their head regions gradually expand in size to become mushroom-shaped. The actin cytoskeleton is a major structural component of both filopodia and mushroom spines (Lei et al., 2016). When spines grow and mature, their increase in surface area requires addition of both lipid and membrane components. These constituents are transported between intracellular membrane organelles and the plasma membrane by a vesicular transport system. However, it is quite unclear how this transport is regulated for dendritic spine formation.

Vesicle transport is controlled by a family of Rab small GTPases. One of these Rabs, Rab11, has been shown to be involved in spine formation (Park et al., 2006; Hoogenraad et al., 2010; Esteves da Silva et al., 2015; Bodrikov et al., 2017). Rab11 is a major regulator of recycling endosomes, which transport endocytosed cell surface receptors back to plasma membrane for reuse (Ullrich et al., 1996; Maxfield and McGraw, 2004). While the role of Rab11 in recycling endosome trafficking is extensively studied in non-neuronal cells, it is not clear yet what roles Rab11 plays in neurons with polarized long processes. Further, it is not known at all how Rab11 is regulated, for example, by guanine nucleotide exchange factors (GEFs) or GAPs in neurons. It is only recently that Rab11A-specific GEFs and GAPs were reported (Dabbeekeh et al., 2007; Fuchs et al., 2007; Gallo et al., 2014; Sakaguchi et al., 2015; Thomas and Fromme, 2016). However, how these regulators function in neurons as well as how they are modulated by upstream signaling cascades remain unaddressed.

Lemur kinase 1A (LMTK1A) is a novel, large Ser/Thr kinase consisting of the N-terminal kinase domain with a long C-terminal tail (Tomomura et al., 2007). LMTK1A was originally identified as a gene upregulated in apoptotic myeloma cells (Gaozza et al., 1997). Since the amino acid sequence of the kinase domain is similar to that of a receptor tyrosine kinase, it was named apoptotic-associated tyrosine kinase (AATYK) (Gaozza et al., 1997). However, it was later shown to be mainly expressed in neurons (Baker et al., 2001; Tomomura et al., 2001) and renamed LMTK1 after the long C-terminal tail. LMTK1 is now considered to be a Ser/Thr kinase, with activity similar to LMTK2 (Kawa et al., 2004; Wang and Brautigan, 2006). We isolated LMTK1 as a p35 Cdk5 activator-binding protein (Honma et al., 2003) and demonstrated its Cdk5-dependent phosphorylation (Tsutsumi et al., 2008). LMTK1A binds to recycling endosomes through palmitoylation of N-terminal cysteines (Takano et al., 2010; Tsutsumi et al., 2010). We recently reported that LMTK1 regulates axon outgrowth and dendrite arborization through Rab11Adependent endosomal trafficking (Takano et al., 2012, 2014). Here, we show that LMTK1 regulates dendritic spine formation through Rab11 activity. Further, we find that TBC1D9B, a Rab11A GAP, also regulates spine formation downstream of
LMTK1. We propose the LMTK1-TBC1D9B-Rab11 signaling pathway as a novel cascade regulating neuronal spine formation via endosomal transport to postsynaptic spines.

\section{Materials and Methods}

Antibodies and plasmids. Anti-GluR2 (clone \#6C4; catalog \#MAB397; RRID:AB_2113875), anti-Myc (clone \#4A6; catalog \#05-724; RRID: AB_11211891), and anti-synaptophysin (clone \#MAB368; catalog \#MAB368; RRID:AB_94947) were purchased from Millipore. Anti-Flag (clone \#M2; catalog \#F3165; RRID:AB_259529), anti- $\beta$-tubulin (clone \#TUB2.1; catalog \#T4026; RRID:AB_477577), and anti-actin (catalog \#A2066; RRID:AB_476693) were obtained from Sigma-Aldrich. AntiGFP (catalog \#11814460001; RRID:AB_390913) was from Roche Diagnostics. Anti-PSD95 (clone \#6G6-1C9; catalog \#MA1-045; RRID; AB_325399), anti-Rab11 (catalog \#71-5300; RRID:AB_2533987), and anti-transferrin (Tf) receptor (clone \#H68.4; catalog \#13-6800; RRID: AB_2533029) were purchased from Thermo Fisher Scientific. Anti-GFP (catalog \#598; RRID:AB_591819) was purchased from MBL. Control IgG (catalog \#sc-2025) was obtained from Santa Cruz Biotechnology. Secondary antibody conjugated with AlexaFluor-488 (catalog \#A-11029; RRID: AB_2534088) and AlexaFluor-647 (catalog \#A-21235; RRID:AB_2535812) was purchased from Invitrogen. Swine anti-rabbit immunoglobulins/HRP (catalog \#P0399; RRID:AB_2617141) and goat anti-mouse immunoglobulins/HRP (catalog \#P0447) were from Dako.

The knockdown vectors of LMTK1 miRNA (miR-LMTK1) and control scramble miRNA-SC (miR-Scr) were constructed into pSUPER. puro (Oligoengine) (Takano et al., 2012). Lifeact-mCherry was provided by Dr. K. Mizuno (Tohoku University) (Riedl et al., 2008). The expression plasmids encoding LMTK1A-Flag wt and its kinase-negative $(\mathrm{kn})$ mutant D206V were described previously (Takano et al., 2012). For expression in neurons, pCAG-LMTK1A-EGFP wt was constructed by inserting the coding region of LMTK1A wt or kn in pEGFP-N1 (Clontech Laboratories) with an EGFP tag at the C-terminal, with subsequent insertion into a pCAG-MCS2 vector (Kawauchi et al., 2005). pEGFPRab11A was previously described (Tsuboi and Fukuda, 2006). pCAGEGFP-Rab11A was constructed by inserting EGFP-Rab11A into a pCAG-MCS2 vector. pEGFP-TBC1D9B and GSTRabin8 were previously described (Itoh et al., 2011; Homma and Fukuda, 2016) An inactive form of TBC1D9B 3A was constructed by mutating Arg559, Tyr592, and Gln594 to Ala (RYQ/AAA) (Gallo et al., 2014) using pEGFP-TBC1D9B as the template. 5'-GTGCTCGGGCATGGAGGCGTGCAGGTCTCGCTC-3' and 5'-GAGCGAGACCTGCACGCCTCCATGCCCGAGCAC-3' were used as forward and reverse primers for Arg559 mutation; 5' -CCCCA CCATCGGCGCCTGCGCGGCAATGAACATC-3' and 5' -GATGTTCA TTGCCGCGCAGGCGCCGATGGTGGGG-3' were forward and reverse primers for Tyr592 and Glu594 mutations. pCAG-EGFP-TBC1D9B was constructed by inserting EGFP-TBC1D9B into pCAG-MCS2 vector for transfection into neurons. Mouse TBC1D9B (NM_029745) tagged with Myc-DDK (MR211851) was purchased from Origene. The cDNAs of SH3BP5, Trappc2l, and Trappc4 were cloned from a cDNA library prepared from mouse primary neurons by PCR. $5^{\prime}$-AGCTTGGTACCGAGC TCGATGGACACGGCGCTGAAACG- ${ }^{\prime}$ and $5^{\prime}$-CACACTGGACTAGT GGATGCCAATCTGCACCATTTTTATGTCAGCAAT-3' were $5^{\prime}$-terninal forward and $3^{\prime}$-terminal reverse primers, respectively, for SH3BP5 cDNA; 5'-AGCTTGGTACCGAGCTCGATGGCGGTGTGCATCGCGGTGATCG CCAAG- ${ }^{\prime}$ and $5^{\prime}$-CACACTGGACTAGTGGAGCACACCTGGATCATC ATGGAAGTCACCAT- $3^{\prime}$ were $5^{\prime}$-terminal forward and $3^{\prime}$-terminal reverseprimers, respectively, forTrappc2lcDNA; $5^{\prime}$-AGCTTGGTACCGA GCTCGATGGCGATTTTTAGCGTGTACGTGGTGAAC-3' and 5'-CC ACACTGGACTAGTGGATGACCCAGGTCCAAAAGTGCCAGCCT TCTC- 3 ' were $5^{\prime}$-terminal forward and $3^{\prime}$-terminal reverse primers, respectively, for Trappc4 cDNA. Cloned cDNAs were subcloned into pcDNA3.1/myc-his (Invitrogen). The mouse TBC1D9B knockdown shRNA sequences were $5^{\prime}$-GGTGACCATTGTTGAAAAAGCTTGGAT CCAGCTTTTTCAACAATGGTCACC-3' for shTBC1D9B \#1, 5' -CTGA CTTCCTCCAGAAAACGCTTGGATCCAGCGTTTTCTGGAGGAAGT CAG-3' for shTBC1D9B \#2, 5'-GACCCCAGCCTGCCCTACCTGTT GGATCCACAGGTAGGGCAGGCTGGGGTC-3' for shTBC1D9B \#3, 
and 5'-GCCATTTAACAAGCTACATATTTGGATCCAATATGTAGC TTGTTAAATGGC-3' for shTBC1D9B \#4. The knockdown sequences were inserted into a pSUPER.puro vector. The scramble control vector was previously described (Furusawa et al., 2017). For RT-PCR detection of TBC1D9B expression in mouse brain, DNA sequence of 5'-24 TAA GAACATCTCAGCTCTAAAGCGA- ${ }^{\prime}$ and 5' -GGCAAAGCAGATGTA GTTATTGGAG-3' was used as $5^{\prime}$-terminal forward and $3^{\prime}$-terminal reverse primers using a cDNA library prepared from mouse primary neurons as a template.

Knockdown vector-resistant LMTK1A (LMTK1 Res) was constructed by mutating eight nucleotides within the knockdown sequence in pcDNA3.1-LMTK1A-myc-His by PCR-based site-directed mutagenesis. First, three nucleotides were changed using GAATCCTGGGATCCCG GCTACGTTAGTAGCTTCACAGACAG and CTGTCTGTGAAGCTAC TAACGTAGCCGGGATCCCAGGATTC as primers, then two nucleotides were changed using CCTGGGATCCCGGTTACGTCAGTAGCTTCACAG and CTGTGAAGCTACTGACGTAACCGGGATCCCAGG as primers, and finally three nucleotides were changed using CGGTTACGTCAGTAGT TTTACCGACAGCTACAG and CTGTAGCTGTCGGTAAAACTACTG ACGTAACCG as primers. After digestion with HindIII and PmeI, the LMTK1A Res-myc was inserted into a pCAG-MC2 vector.

Cell culture and transfection. Neuro-2A (IFO50071; RRID:CVCL_0470), HEK293 (JCRB9068; RRID:CVCL_0045), and COS-7 (JCRB9127; RRID; CVCL_0224) cells were obtained from the JCRB Cell Bank. Neuro-2A cells were maintained in MEM (Thermo Fisher Scientific) supplemented with 10\% FBS (Biowest), 1\% nonessential amino acids (Wako), $50 \mathrm{IU} / \mathrm{ml}$ penicillin, and $50 \mu \mathrm{g} / \mathrm{ml}$ streptomycin as described previously (Tsutsumi et al., 2010; Sharma et al., 2016). They were transfected with indicated plasmids using Lipofectamine 2000 (Invitrogen; catalog \#11668027).

Mice and mouse brain cortical and hippocampal neuron culture. All mouse experiments were performed according to the guidelines for animal experimentation of Tokyo Metropolitan University (approval number A28-10, A29-20). Pregnant ICR mice (RRID:IMSR_TAC:icr) were obtained from Sankyo Laboratory Service. The LMTK1 KO mouse was described previously (Takano et al., 2012). Mice were housed in a temperature-controlled room under a $12 \mathrm{~h}$ light/12 h dark cycle with free access to food and water. All efforts were made to reduce animal suffering.

Cerebral cortical and hippocampal neurons were prepared from brains of mouse embryos at embryonic day 16 (E16) or E17, and maintained in neurobasal medium (Invitrogen), 2\% (v/v) B27 (Invitrogen), and $0.5 \mu \mathrm{M}$ L-glutamine (Takano et al., 2014). Transfection was performed with the indicated plasmids using Lipofectamine 2000 at 7, 10, or 14 DIV. In low density culture experiments, neurons were plated on precultured 2 DIV glial cells. Neurons were observed under a confocal laser microscope LSM710 (Carl Zeiss), and images were captured at 0.44 $\mu \mathrm{m}$ intervals for $z$-stack series. Fluorescent intensity was analyzed using ZEN software.

In utero electroporation and tissue processing. Plasmid vectors, miRLMTK1 or miR-Scr in a pSUPER.puro vector $(3 \mu \mathrm{g} / \mu \mathrm{l})$, or LMTK1A wt or $\mathrm{kn}$ in a pCAGGS vector $(1.5 \mu \mathrm{g} / \mu \mathrm{l})$, were injected with pCAGGSEGFP $(1 \mu \mathrm{g} / \mu \mathrm{l})$ into the lateral ventricles of ICR mouse brains at E14 by in utero electroporation using a CUY21 electroporator (Nepa Gene) with $50 \mathrm{~ms}$ of $35 \mathrm{~V}$ electronic pulse for 5 times with $450 \mathrm{~ms}$ intervals (Tabata and Nakajima, 2001). Brains of injected mouse, either sex, were fixed in $4 \%(\mathrm{w} / \mathrm{v})$ PFA in PBS by terminal perfusion at P20. They were then dissected and further fixed overnight at $4^{\circ} \mathrm{C}$. After changing the solution to $15 \%$ sucrose (w/v) in PBS and then $30 \%$ sucrose (w/v) in PBS, cerebral cortex was cut into 50 - to 100 - $\mu \mathrm{m}$-thick sections and observed under a confocal laser microscope Exciter (Carl Zeiss).

Immunofluorescent staining. Primary hippocampal neurons in cultures were fixed with $4 \%$ PFA in PBS for 20 min at $37^{\circ} \mathrm{C}$. After treatment with $0.1 \%$ Triton X-100 and 5\% skim milk in PBS, neurons were probed with antiPSD-95 (1:150) overnight at $4^{\circ} \mathrm{C}$ or anti-synaptophysin (1:250) at room temperature for $1 \mathrm{~h}$ and then incubated with the corresponding secondary antibody (1:250) for $1 \mathrm{~h}$. For analyzing the expression of GluR2, neurons were probed with anti-GluR2 (1:200) at room temperature for $1 \mathrm{~h}$, followed by staining with the indicated secondary antibody (1:250) for $1 \mathrm{~h}$.

Brains of $L M T K 1^{+/+}$or $L M T K 1^{-1-}$ mice of either sex were fixed in $4 \%$ PFA in PBS by terminal perfusion at P21, followed by dissection and
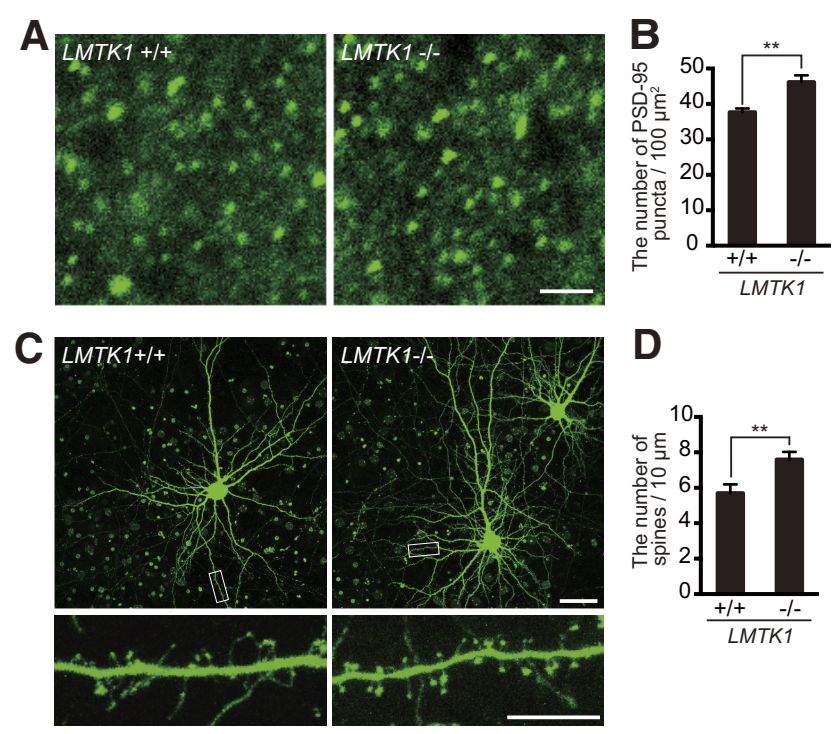

\section{D}

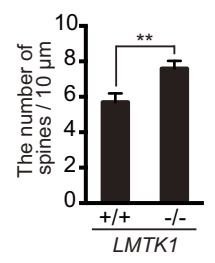

Figure 1. The density of PSD-95-positive puncta in cerebral cortex of LMTK1 ${ }^{-1-}$ mouse brain. $\boldsymbol{A}$, Immunostaining of cerebral cortex of $L M T K 1^{+1+}$ (left) or $L M T K 1^{-1-}$ (right) mouse brain at P21 with anti-PSD-95. Scale bar, $2 \mu \mathrm{m}$. $\boldsymbol{B}$, The number of PSD-95-positive puncta per $100 \mu \mathrm{m}^{2}$ (mean \pm SEM, 6 cortical areas analyzed from two mice each for $L M T K 1^{+1+}$ and

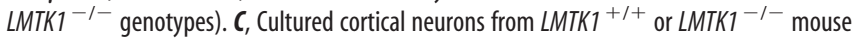
brains. Primary cortical neurons were transfected with GFP at 15 DIV to visualize dendritic spines and observed at 18 DIV. Bottom, Higher-magnification images of a dendrite, demarcated with a box in the top. Scale bars: top, $50 \mu \mathrm{m}$; bottom, $10 \mu \mathrm{m}$. $D$, The density of spines shown as mean $\pm \operatorname{SEM}\left(n=10\right.$ for both $L M T K 1^{+/+}$and $\left.L M T K 1^{-/-}\right){ }^{* *} p<0.01$.

further fixation overnight at $4^{\circ} \mathrm{C}$. After changing the solution to $15 \%$ sucrose in PBS, and then $30 \%$ sucrose in PBS, cerebral cortex was cut into $10-\mu \mathrm{m}$-thick sections. Samples were treated with $0.1 \%$ Triton X-100 and $5 \%$ BSA in PBS, and then probed with anti-PSD-95 (1:100) overnight at $4^{\circ} \mathrm{C}$, followed by incubation with the corresponding secondary antibody (1:250).

Tf uptake and recycling assay. Tf uptake and recycling assays were performed as described previously (Takano et al., 2010). Briefly, for the Tf uptake assay, after incubation in serum-free medium for $1 \mathrm{~h}$ at $37^{\circ} \mathrm{C}$, COS-7 cells or primary neurons were incubated in ice-cold medium containing $10 \mu \mathrm{g} / \mathrm{ml}$ AlexaFluor-546-conjugated Tf (Alexa-Tf) (T23364, Thermo Fisher Scientific) for $30 \mathrm{~min}$ at $4^{\circ} \mathrm{C}$ and then warmed at $37^{\circ} \mathrm{C}$ for $30 \mathrm{~min}$. For the Tf recycling assay, primary neurons, into which LMTK1 wt-EGFP or kn-EGFP was transfected using an Amaxa Nucleofector apparatus (Lonza), were used. Primary neurons at 3 DIV were incubated in neurobasal medium containing $10 \mu \mathrm{g} / \mathrm{ml}$ Alexa-Tf at $37^{\circ} \mathrm{C}$ for $60 \mathrm{~min}$. After incubation with $100 \mu \mathrm{g} / \mathrm{ml}$ unlabeled Tf (Sigma-Aldrich) at $37^{\circ} \mathrm{C}$ for 10 and $30 \mathrm{~min}$, neurons were fixed with $4 \%$ PFA. The number of Alexa-Tf particles $(>0.01 \mu \mathrm{m})$ in the cell body of neurons expressing LMTK1A wt-EGFP or kn-EGFP was counted using ImageJ software after digital images were acquired with an LSM710.

Live cell imaging. Primary hippocampal neurons were plated on glassbottom dishes (Greiner Bio-One) coated with polyethylenimine, and were cultured using Neurobasal medium (Invitrogen) supplemented with B-27. The medium was changed to Neurobasal without phenol red supplemented with B-27, and fluorescent images were captured at every 2 min for 10 min under an LSM710 confocal microscope.

Immunoprecipitation. Neuro-2a cells transfected with the indicated plasmids were disrupted by sonication in lysis buffer, $50 \mathrm{~mm}$ Tris- $\mathrm{HCl}$, pH 7.5, 0.5\% Nonidet P-40, 10\% glycerol, $150 \mathrm{~mm} \mathrm{NaCl}, 1$ mm EDTA, 2.5 mм $\mathrm{MgCl}_{2} 1 \mathrm{~mm} \mathrm{NaF}, 0.1 \mathrm{~mm} \mathrm{Na}_{3} \mathrm{VO}_{4}, 1$ mм DTT, 0.4 mм Pefabloc, 1 mм $\beta$-glycerophosphate or 50 mм Tris- $\mathrm{HCl}$, pH 7.5, $100 \mathrm{~mm} \mathrm{NaCl}, 1 \mathrm{~mm}$ EDTA, 0.5\% Triton X-100, 1 mm NaF, 1 mм DTT, 0.4 mm Pefabloc, 1 mm $\beta$-glycerophosphate. After centrifugation at $14,000 \times g$ for $15 \mathrm{~min}$, the supernatant was incubated with anti-Flag or control IgG for $90 \mathrm{~min}$ on ice. After addition of Protein-G Sepharose beads (GE Healthcare BioScience), they were further incubated for $90 \mathrm{~min}$ at $4^{\circ} \mathrm{C}$. After washing 
with lysis buffer, proteins bound to beads were eluted with Laemmli's sample buffer.

Cell fractionation. Neuro-2a cells transfected with indicated plasmids were disrupted by passing through a 27-gauge needle in $20 \mathrm{~mm}$ HEPES, pH 7.5, $100 \mathrm{~mm} \mathrm{NaCl}, 2 \mathrm{~mm} \mathrm{MgCl}_{2}, 1$ mм EDTA, 0.4 mм Pefabloc, $10 \mu \mathrm{g} / \mu \mathrm{l}$ leupeptin, $10 \mathrm{~mm} \mathrm{NaF}, 10 \mathrm{~mm} \beta$-glycerophosphate, and $1 \mathrm{mM} \mathrm{Na}_{3} \mathrm{VO}_{4}$. After centrifugation at $1100 \times g$ for $4 \mathrm{~min}$ at $4^{\circ} \mathrm{C}$, the supernatants were centrifuged at $100,000 \times g$ for $1 \mathrm{~h}$ to separate the membrane and cytosolic fractions.

GST pulldown assay of active GTP-bound Rab11a. GST-Rabin8 was expressed in Escherichia coli BL21(DE3) and bound to GSHSepharose beads. HEK293 cells were transfected with EGFP-Rab11A, TBC1D9B-Myc and empty vector, LMTK1A-Flag wt or $\mathrm{kn}$ using Lipofectamine 2000. The cells were disrupted in 50 $\mathrm{mm}$ Tris-HCl, $\mathrm{pH} 7.5,0.5 \%$ Triton X-100, $150 \mathrm{~mm} \mathrm{NaCl}, 1 \mathrm{~mm} \mathrm{MgCl}_{2}, 10 \mathrm{~mm} \mathrm{NaF}, 0.1$ mM $\mathrm{Na}_{3} \mathrm{VO}_{4}, 1 \mathrm{~mm}$ DTT, $0.4 \mathrm{~mm}$ Pefabloc, $10 \mu \mathrm{g} / \mathrm{ml}$ leupeptin, and $10 \mathrm{~mm} \beta$-glycerophosphate. The cell extract obtained by centrifugation at $15,000 \times g$ for $15 \mathrm{~min}$ at $4^{\circ} \mathrm{C}$ was incubated with GST-Rabin8 bound to GSH beads at $4^{\circ} \mathrm{C}$ for $1 \mathrm{~h}$. After washing with the buffer, Rab11A bound to the GSH-Sepharose beads was detected by immunoblotting with anti-Rab11 (1:2000).

SDS-PAGE and immunoblotting. SDS-PAGE was performed with $5 \%, 7.5 \%, 10 \%$, or $12.5 \%$ polyacrylamide for separating gels. Immunoblotting was performed as described previously (Furusawa et al., 2017). The primary antibodies used were anti-myc (1:2000), anti-GFP (1:1000), anti-Tf receptor $(1: 1000)$, anti- $\beta$ tubulin (1:1000), anti-Flag (1:1000), and anti-actin (1:2000).

Agarose gel electrophoresis. Agarose gel electrophoresis was performed with $1 \%$ agarose gel, and DNAs were stained by GRRed Loading Buffer (GRR-1000, Bio Craft).

Quantification and statistical analysis. Band intensities of immunoblots were estimated by ImageJ (https://imagej.nih.gov/ij/). Spine densities were measured by counting the number of spines on a $10 \mu \mathrm{m}$ section of a dendritic shaft using Photoshop software (Adobe). One section was chosen from each neuron. Statistical analyses were performed using Prism7 software (GraphPad; RRID:SCR_002798). A Student's $t$ test was used to analyze the number of PSD-95 puncta in Figure $1 B$, spine density in Figures $1 D$ and $2 B, G$, PSD-95- or GluR2-positive spine (\%) and density, synaptic spines (\%), synapse density in Figure $3 C, D, F, G, I, J$, and frequency of spine formation and elimination in Figure $5 B, C$. A one-way ANOVA followed by Tukey's multiple-comparisons test was used to analyze the spine density in Figures $4 B, D, 7 B$, and $9 E, G, I$, and data of immunoblots in Figure $8 B, G$. The spines were classified into four types depending their morphology. Criteria are as follows: filopodia, spines without a head; stubby, spines without a neck; atypical, spines with irregular shape; and mushroom, spines with a head and a neck (Ultanir et al., 2012). Their percent ratios were analyzed by two-way ANOVA with repeated measurements, followed by a Bonferroni correction. Spine density in Figures $7 E$ and $9 K$ was analyzed by a two-way ANOVA, followed by Tukey's multiple-comparison. Post hoc analyses were performed when there was a significant interaction to determine the simple main effect. All data are expressed as mean \pm SEM. Significance is shown, and not significant values are not noted.

\section{Results}

The density of spines is increased in LMTK1-deficient mouse brains and neurons

Previous studies indicate that expression of LMTK1 is upregulated during neuronal development in vivo (Tomomura et al., 2007), and LMTK1 regulates the transport of Rab11A-positive endosomes in axons and dendrites (Takano et al., 2012, 2014). Further, Rab11A is reported to have a role in dendritic spine formation (Park et al., 2006; Hoogenraad et al., 2010; Bodrikov et al., 2017). Therefore, we hypothesized that LMTK1 regulates spine formation by modulating Rab11A activity. To address this question, we compared the number of spines in cerebral cortex from brains of $L M T K 1^{+/+}$and LMTK1 $^{-1-}$ mice at P21, when spines are established in vivo. We immunostained for the excitatory postsynaptic scaffold protein, PSD-95, a common marker of mature spines. The number of PSD95-positive puncta was greater in the brains of $L M T K 1^{-1-}$ mice than in $L M T K 1^{+/+}$mice, suggesting an increase in spine number with decreased LMTK1 expression (Fig. 1 A, B; $p=0.0031$, Student's $t$ test). Next, we confirmed that dendritic spines are indeed increased 


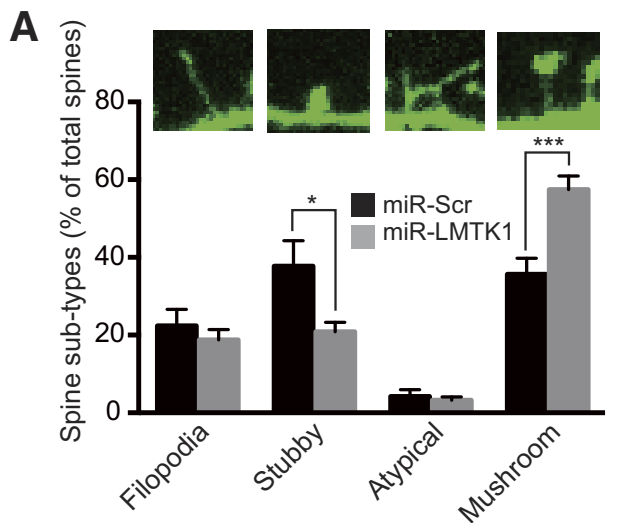

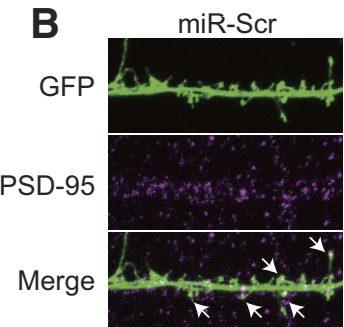

miR-LMTK1
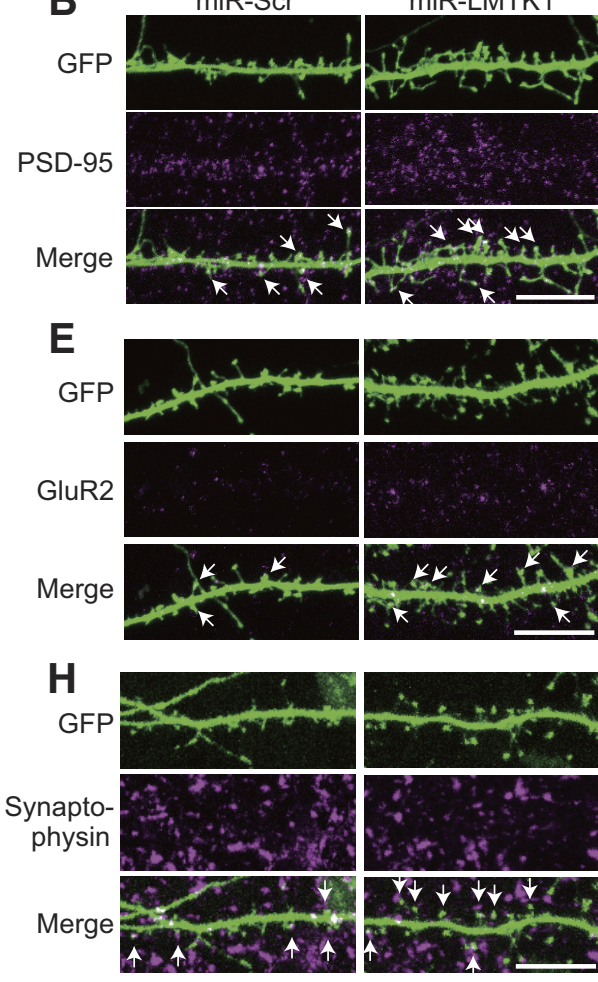

C

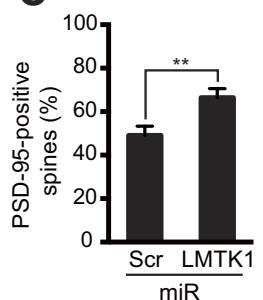

F

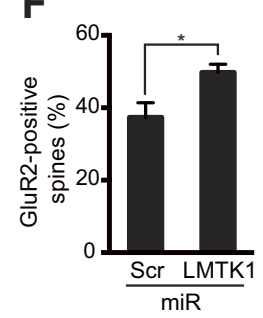

I

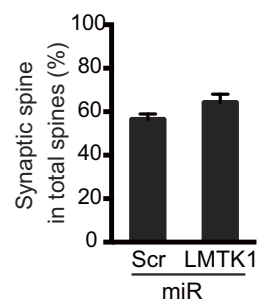

Figure 3. Knockdown of LMTK1 leads to an increase in mature spine morphology. $A$, The classification of spines and corresponding ratios. Spines are classified into filopodia, stubby, atypical, and mushroom depending on morphology (Ultanir et al., 2012). Top, Representative images. Their ratios are expressed as mean \pm SEM ( $n=20$ neurons for miR-Scr; $n=35$ for miR-LMTK1). $\boldsymbol{B}$, PSD-95-positive spines in LMTK1 knockdown neurons. Hippocampal neurons were transfected with GFP and miR-LMTK1 or miRScr at 7 DIV and spines were observed after staining with anti-PSD-95 (middle) at 14 DIV. Bottom, Merge of GFP and PSD-95 staining. Scale bar, $10 \mu \mathrm{m} . C, D$, The ratio and number of PSD-95-positive spines are expressed as mean \pm SEM ( $n=9$ for miR-Scr; $n=8$ for miR-LMTK1). $E$, GluR2 on the spine surface of LMTK1 knockdown neurons. Hippocampal neurons were transfected with GFP and miR-LMTK1 or miR-Scr at 7 DIV, and were immunostained with an antibody against the outer cellular domain of GluR2 at 14 DIV. $\boldsymbol{F}, \boldsymbol{G}$, The ratio and number of GluR2-positive spines are expressed as mean \pm SEM ( $n=8$ for miR-Scr and miR-LMTK1). $\boldsymbol{H}$, Synapse formation visualized by staining of synaptophysin. Hippocampal neurons were transfected with GFP and miRLMTK1 or miR-Scr at 7 DIV, and synapse formation was examined by immunostaining for the presynaptic protein, synaptophysin (middle), at 14 DIV. Bottom, Merges of GFP and synaptophysin staining. Scale bar, $10 \mu \mathrm{m}$. I, J, The ratio and number of spines with synaptophysin staining are indicated as mean \pm SEM ( $n=7$ for miR-Scr, $n=7$ for miR-LMTK1). ${ }^{*} p<0.05,{ }^{* *} p<0.01,{ }^{* * *} p<0.001$.

in neurons of $L M T K 1^{-1-}$ mice using primary neuronal cultures. Cultured neurons prepared from brains of $L M T K 1^{+/+}$or $L M T K 1^{-1-}$ mice were transfected with GFP for visualization of dendritic spines. At $18 \mathrm{DIV}$, the density of spines was higher in LMTK1 ${ }^{-1-}$ neurons than in $L M T K 1^{+/+}$neurons (Fig. $1 C, D ; p=$ 0.0094 , Student's $t$ test). These results suggest that LMTK1 plays a role in spine formation.
D

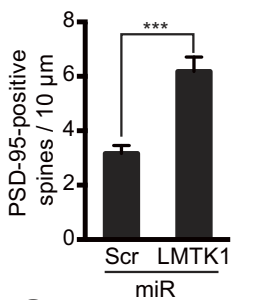

G

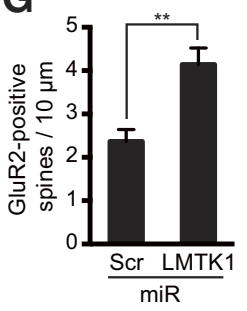

J

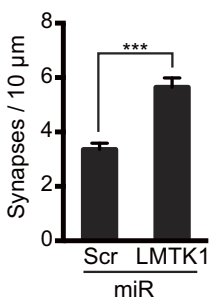

The effect of LMTK1 knockdown on dendritic spine formation

Dendrite outgrowth is stimulated in LMTK $1^{-1-}$ neurons (Takano et al., 2014). Therefore, it is possible that the observed increase in spine formation is a secondary effect of enhanced dendrite outgrowth. To ask whether LMTK1 functions directly in spine formation, we knocked LMTK1 down in hippocampal neurons with miR-LMTK1 (Takano et al., 2012, their Fig. 7A,\#3) at 7 DIV, after dendrites have extended, and cotransfected with GFP to enable spine visualization and analysis at 18 DIV (Fig. 2A). The knockdown of LMTK1 increased spine density by $36 \%$ over the scrambled micro RNA (miR-Scr) control (Fig. $2 B ; p<$ 0.0001 , Student's $t$ test).

We confirmed the specificity of miRLMTK1 by cotransfection of a knockdown vector-resistant LMTK1 Res. Expression of LMTK1-Res was evident in the presence of miR-LMTK1, whereas the expression of LMTK1 WT was suppressed (Fig. 2C). The density of spines was reversed to the level of a control experiment with miRScramble by coexpression of LMTK1 Res with miR-LMTK1 (Fig. $2 D, E ; F_{(2,36)}=$ 12.45 for one-way ANOVA, $p<0.0001$ for miR-Scramble vs miR-LMTK1, $p<$ 0.0001 for miR-LMTK1 vs LMTK1 Res with miR-LMTK1, Tukey's multiplecomparisons test).

Next, we evaluated the effect of LMTK1 knockdown on spine formation in vivo in developing brain using in utero electroporation (Tabata and Nakajima, 2001). A knockdown vector of LMTK1 was introduced into cortical neurons at embryonic day 14 (E14), and spines were observed at postnatal day 20 (P20), when all neurons have completed their migration to cortical layers. In layer $2 / 3$ of the cortex, we found that knockdown of LMTK1 increased neuronal spine density (Fig. $2 F, G ; p=$ 0.0004 , Student's $t$ test), indicating that LMTK1 regulates spine formation in vivo in developing neurons.

\section{LMTK1 regulates spine maturation}

Spines are classified into four different types, filopodia, stubby, atypical, and mushroom, depending on the stage of spine formation (as described in Materials and Methods; Fig. 3A, top) (Ultanir et al., 2012). To determine where LMTK1 works in this process, we evaluated spine morphology in LMTK1-knockdown neurons. While all four spine types were found in LMTK1-knockdown neurons, the population of the mushroom-type spines was significantly increased in LMTK1 knockdown neurons compared with control neurons (Fig. 3A; $p<0.0001$ for two-way ANOVA with repeated measurement, $57 \%$ vs $35 \%$ for Mushroom-miR- 
A
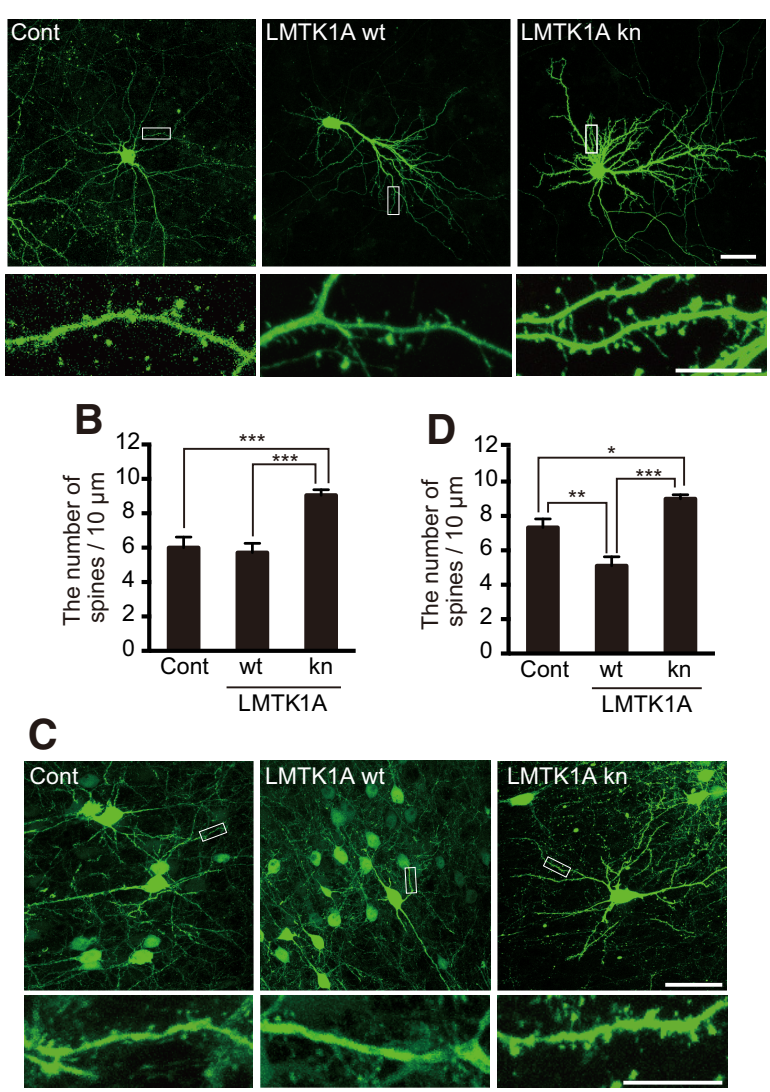

Figure 4. The role of LMTK1A kinase activity in dendritic spine formation. $\boldsymbol{A}$, The effect of kn LMTK1A on spine formation in vitro. Hippocampal neurons were transfected with GFP and an empty vector (Cont), LMTK1A wt, or kn at 7 DIV and dendritic spines were observed at 18 DIV. Bottom, Higher magnification of the box indicated in top. Scale bars: top, $50 \mu \mathrm{m}$; bottom, 10 $\mu \mathrm{m} . B$, Quantification of the spine density (mean $\pm \mathrm{SEM}, n=10$ for Control; $n=14$ for LMTK1A wt; $n=20$ for kn). $C$, The effect of LMTK1A on spine formation in vivo. Neural progenitors were transfected with GFP and an empty vector (Cont) (left), LMTK1A wt (middle), or kn (right) at E14, and dendritic spines were observed at P20. Bottom, Higher magnification of the box indicated in top. Scale bars: top, $50 \mu \mathrm{m}$; bottom, $10 \mu \mathrm{m}$. D, Spine densities are shown as mean \pm SEM ( $n=12$ for control; $n=10$ for LMTK1A wt; $n=11$ for kn). ${ }^{*} p<0.05,{ }^{* *} p<$ $0.01,{ }^{* * *} p<0.001$.

LMTK1 vs Mushroom-miR-Scr, $p=0.0011$, Bonferroni's multiple correction), suggesting that LMTK1 regulates the maturation, as well as the number of spines.

We next examined the structural maturity of mushroom spines in LMTK1-knockdown neurons by immunostaining with anti-PSD-95 at 14 DIV (Fig. 3B). PSD-95 was detected in most of the mushroom spines, and the ratio of PSD-95-positive spines was higher in LMTK1 knockdown neurons than control neurons ( $p=0.0095$ for Fig. $3 C$; $p<0.0001$ for Fig. 3D; Student's $t$ test). Another hallmark of spine maturation is the presence of AMPA receptors in the postsynaptic membrane. When we immunostained for the AMPA receptor extracellular domain, GluR2 (Fig. $3 E$ ), we found that the ratio and number of GluR2-positive spines were increased in LMTK1 knockdown neurons compared with control neurons ( $p=0.0094$ for Fig. $3 F ; p=0.0021$ for Fig. $3 G$; Student's $t$ test). Moreover, the intensity of GluR2 was increased by $\sim 43 \%$ in the dendrite surface of LMTK1 knockdown neurons over control neurons ( $n=8$ for miR-Scr; $n=9$ for miR-LMTK1, $p=0.003$, Student's $t$ test). Finally, synapse formation was validated by immunostaining for the presynaptic protein synaptophysin at 14 DIV (Fig. $3 H$ ). The number of spines with juxtastaining of synaptophysin was increased in neurons with LMTK1
A
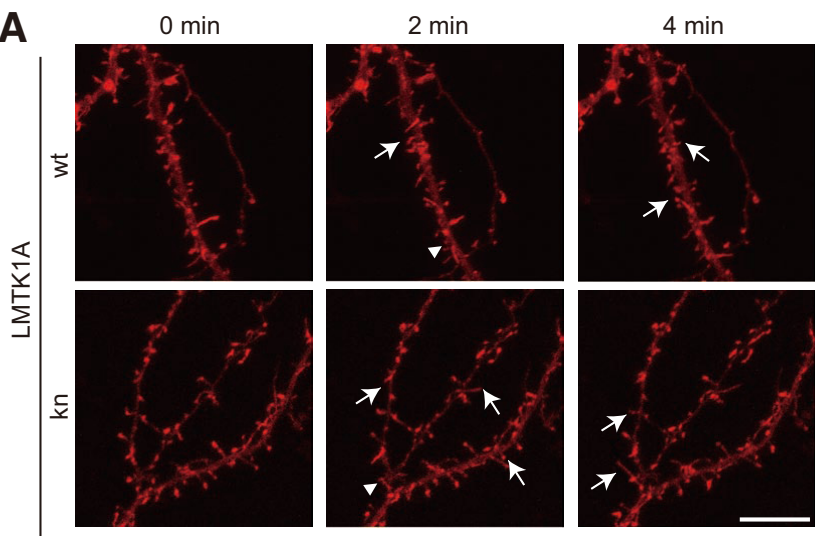

B

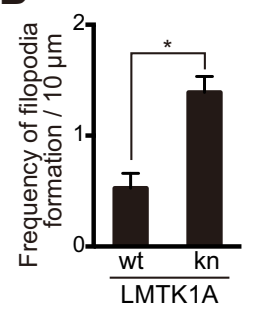

C
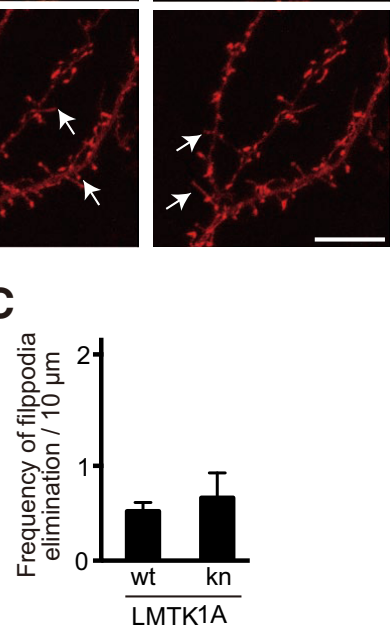

Figure 5. Dynamics of filopodial protrusions on dendrites. A, Hippocampal neurons were transfected with Lifeact-mCherry and LMTK1A-GFP wt (top) or kn (bottom) at 13 DIV. Dendritic filopodial protrusions were observed with live imaging of DIV16 neurons expressing Lifeact$\mathrm{mCherry}$ (imaged every $2 \mathrm{~min}$ for a total of $10 \mathrm{~min}$ ). Still images at 0, 2, and $4 \mathrm{~min}$ are shown. Arrows indicate formation of protrusions. Arrowheads indicate elimination of protrusions. Scale bar, $10 \mu \mathrm{m} . \boldsymbol{B}, \boldsymbol{C}$, The frequency of formation and elimination is indicated as mean $\pm \mathrm{SEM}(n=$ 8 for LMTK1A wt; $n=4$ for kn). ${ }^{*} p<0.05$.

knocked down (Fig. 3I,J; $p=0.0001$, Student's $t$ test). These results indicate that LMTK1 negatively regulates spine formation and maturation.

\section{LMTK1A regulates dendritic spine formation through its kinase activity}

LMTK1 is considered to be a Ser/Thr kinase, but its kinase activity has not been directly demonstrated. Indeed, it is possible that LMTK1 serves as a scaffold protein rather than a kinase because several signaling proteins, such as p35 Cdk5 activator, Ste-20related proline-alanine-rich kinase, and protein phosphatase $1 \mathrm{C}$, are reported to bind its long C-terminal tail (Honma et al., 2003; Gagnon et al., 2007; Manser et al., 2012). To test whether the kinase activity of LMTK1 is required for regulation of spine formation, we expressed a D206V kn mutant of LMTK1A (Fig. 4A). LMTK1A is an isoform of LMTK1, which binds to recycling endosomes through palmitoylation of three cysteines in the N-terminal region (Tsutsumi et al., 2008). Asp206 is a conserved amino acid in the kinase domain, which is required for activity of many protein kinases, and its mutation to Val (D206V) has been used as a kn construct (Tomomura et al., 2001). The dendritic spine density was significantly increased in neurons expressing LMTK1A kn compared with control neurons (Fig. $4 A, B ; F_{(2,41)}=18.4$ for one-way ANOVA, $p=0.0002$ for Cont vs kn, Tukey's multiplecomparisons test). Overexpression of wt LMTK1A did not change the number of spines, as was previously shown with axon and dendrite outgrowth (Takano et al., 2012, 2014). In utero electroporation of LMTK1A kn at E14 also increased the number of spines in vivo when evaluated at P20, after neurons have migrated and formed synapses in cortical layers (Fig. $4 C, D ; F_{(2,30)}=$ 


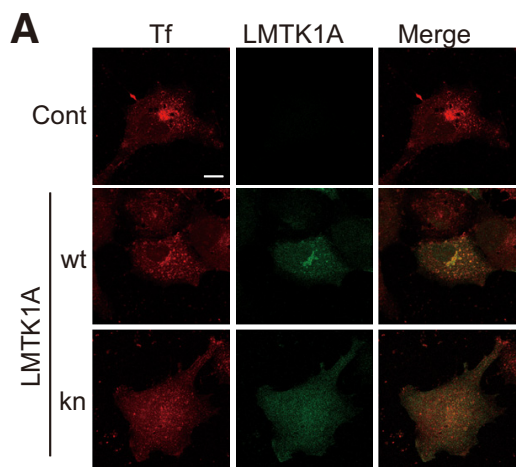

B
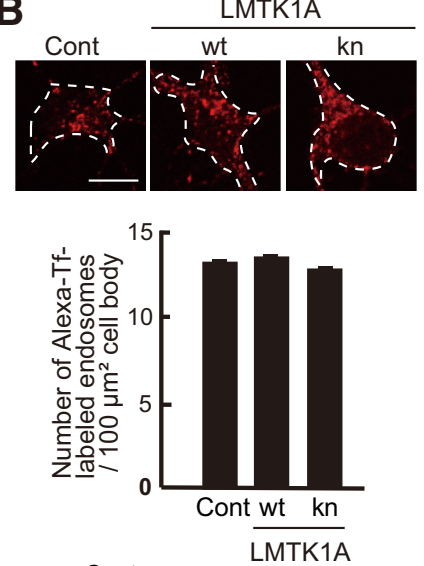

C

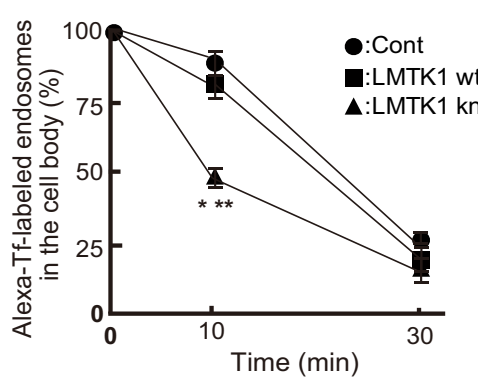

Figure 6. Effect of LMTK1A kn on cellular distribution and transport of Tf-labeled endosomes. A, Localization of internalized AlexaFluor-546-If (Alexa-Tf) in COS-7 cells, which were transfected with LMTK1A wt-EGFP, LMTK1A kn-EGFP, or empty vector. Left, Alexa-Tf. Middle, LMTK1A immunostaining. Right, Merges. Scale bar, $10 \mu \mathrm{m}$. B, Alexa-Tf internalized into primary neurons. Cerebral cortical neurons were transfected with LMTK1A wt-EGFP or LMTK1 kn-EGFP at 0 DIV and were incubated with Alexa-If at 3 DIV. Alexa-If in the cell body of neurons labeled with EGFP was observed at 30 min after incubation. Scale bar, $20 \mu \mathrm{m}$. The number of Alexa-Tf-positive endosomes ( $>0.01 \mu \mathrm{m}$ in diameter) were counted and expressed (bottom; mean \pm SEM, $n=10$ for each neuron expressing EGFP (Cont), LMTK1 wt-EGFP, or LMTK1 kn-EGFP). C, The decrease of Alexa-Tf-positive endosomes in the cell body of neurons after chasing. After $1 \mathrm{~h}$ incubation with Alexa-If, neurons were washed and further incubated with neurobasal medium without Alexa-Tf for 10 and $30 \mathrm{~min}$. The number of Alexa-Tf-positive endosomes was counted in the cell body of neurons labeled with EGFP (mean \pm SEM, $n=15$ for each of EFGP (Cont), LMTK1 wt-EGFP, and LMTK1 kn-EGPF). ${ }^{*} p<0.05$ between LMTK1A kn and wt. ${ }^{* *} p<0.01$ between LMTK1A kn and Cont.

18.8 for one-way ANOVA, $p=0.0267$ for Cont vs kn, $p<0.0001$ for wt vs kn, Tukey's multiple-comparisons test). These results indicate that the kinase activity of LMTK1 is involved in spine formation.

\section{Frequency of filopodia formation is increased in neurons} overexpressing LMTK1A kn

Knockdown of LMTK1 or overexpression of LMTK1A kn increased neuronal spine density. Spines are dynamic structures, with continual formation and elimination (Dunaevsky et al., 1999). To determine whether an increase in formation or a decrease in elimination is driving the change in spine density, we observed the dynamics of dendritic protrusions by visualizing F-actin using Lifeact-mCherry expression in living neurons. Still images at 0,2, and 4 min are shown in Figure 5A. Newly formed and eliminated protrusions are indicated by arrows and arrowheads, respectively. Overexpression of LMTK1A kn increased the frequency of filopodial protrusion appearance compared with LMTK1A wt (Fig. 5B; $p=0.0027$, Student's $t$ test). In contrast, the frequency of elimination was not significantly different between the two conditions (Fig. $5 \mathrm{C}$ ). These results suggest that the loss of LMTK1A activity increases spine density by enhancing the formation of spines rather than suppressing their disappearance.

\section{LMTK1 regulates dendritic spine formation via Rab11A}

Recent reports indicate a role for Rab11A in spine formation (Park et al., 2006; Hoogenraad et al., 2010; Takano et al., 2012;
Bodrikov et al., 2017). Before going to the analysis of the LMTK1-Rab11 axis in spine formation, we confirmed the effect of LMTK1 kn on recycling endosome localization using Alexa-Tf. While internalized Alexa-Tf accumulated at the perinuclear region in the cells expressing LMTK1 wt as was observed in control cells expressing EGFP, Alexa-Tf was diffusely distributed in the whole cytoplasm in LMTK1A kn-expressing COS-7 cells (Fig. $6 A)$. Next, we examined the internalization of Alexa-Tf in primary neurons. After 30 min incubation with Alexa-Tf, the number of Alexa-Tf-positive endosomes was counted. The similar number of Alexa-Tfpositive endosomes was counted in the cell body of neurons expressing either LMTK1 wt or kn (Fig. 6B), suggesting that endocytosis was not affected by LMTK1. Efflux of Alexa-Tf-labeled endosomes from the cell body was measured after chasing. The decrease in the number of Alexa-Tf particles was faster in neurons expressing LMTK1A kn than that of neurons expressing LMTK1A wt (Fig. $6 C ; F_{(2,42)}=$ 6.028 for one-way ANOVA, $p=0.0063$ for Cont vs kn; $p=0.0273$ for wt vs $\mathrm{kn}$, Tukey's multiple-comparisons test), confirming the acceleration of Rab11-positive endosomal trafficking in LMTK1A KO neurons (Takano et al., 2014).

To confirm the involvement of Rab11A in spine formation in our experimental paradigm, we transfected the constitutively active form of Rab11A, Q70L, or inactive form of Rab11A, S25N, into cultured neurons at 10 DIV, after dendrite formation, and observed spines at 15 DIV. Rab11A Q70L increased the spine density in neurons over those overexpressing Rab11A wt, whereas the spine density was decreased in neurons when Rab11A S25N was overexpressed (Fig. $7 A, B$; $F_{(2,50)}=21.2$ for one-way ANOVA, $p=0.0008$ for wt vs Q70L; $p=0.0103$ for wt vs $\mathrm{S} 25 \mathrm{~N}$, Tukey's multiple-comparisons test). These results validate that Rab11A plays an active role in spine formation.

Previous work illustrated that LMTK1A is cotransported with Rab11A in axons and dendrites (Takano et al., 2014). Therefore, to determine whether LMTK1A enters into spines with Rab11A, we transfected hippocampal neurons with LMTK1A-Flag and Rab11A-GFP at DIV14, and then immunostained for Flag at 16 DIV for confocal analysis. Indeed, LMTK1A-Flag was found to colocalize with Rab11A-GFP in spines (Fig. 7C). Together with our previous observation (Takano et al., 2014), these results suggest that LMTK1 moves into spines on Rab11A-positive endosomes.

We next examined whether the LMTK1-Rab11A pathway operates in spine formation. We addressed this question by overexpressing Rab11A mutants in LMTK1-knockdown neurons. Knockdown of LMTK1 increased spine density in neurons overexpressing Rab11A wt (Fig. 7D, top, $E ; p=0.0169$ for two-way ANOVA, $p=0.0034$ for miR-Scr-Rab11A wt vs miR-LMTK1Rab11A wt, Tukey's multiple-comparisons test). On the other hand, Rab11A Q70L increased spine density whether LMTK1 was knocked down or not (Fig. $7 D$, middle, $E$; $p=0.0169$, two-way 
ANOVA, miR-Scr-Rab11A wt vs miRScr-Rab11A Q70L, $p=0.0014$; miR-ScrRab11A Q70L vs miR-LMTK1-Rab11A Q70L, $p=0.9999$; Tukey's multiplecomparisons test). Similarly, Rab11A S25N decreased spine density regardless of LMTK1 expression (Fig. 7D, bottom, E; miR-Scr-Rab11A wt vs miR-Scr-Rab11A S25N, $p=0.005$ l; miR-Scr-Rab11A S25N vs miR-LMTK1-Rab11A S25N, $p=$ 0.8869, Tukey's multiple-comparisons test). These results clearly indicate that Rab11A functions downstream of LMTK1 in spine formation.

LMTK1A binds to TBC1D9B, a GTPaseactivating protein (GAP) for Rab11A

The above results suggested that Rab11A is more active when LMTK1 is downregulated. Since the active form of Rab11A binds to recycling endosomes, we asked whether LMTK1A alters the amount of Rab11A bound to membrane vesicles. Membrane vesicles were prepared from Neuro-2a cells expressing LMTK1A wt or $\mathrm{kn}$. The successful separation of membrane and cytosolic fractions was confirmed using the respective marker proteins, Tf receptor for the membranes and tubulin for the cytosolic fraction (Fig. $8 A$ ). Approximately half of transfected Rab11A was detected in the membrane fraction, and the expression of LMTK1A $\mathrm{kn}$ increased the amount of Rab11A in the membrane fraction, whereas LMTK1A wt did not (Fig. $8 B ; F_{(2,6)}=25.32$, one-way ANOVA, $p=0.0044$ for $(-)$ vs $\mathrm{kn}, p=$ 0.0012 for wt vs kn, Tukey's multiple-comparisons test). This result suggested that LMTK1 kn increases the GTP-bound active form of Rab11A probably by modulating a GTP-GDP cycle.

How LMTK1 regulates Rab11A activity has remained an open question. Since previous work found no direct interaction between LMTK1 and Rab11A (Takano et al., 2012), we assumed that an unidentified factor mediates the action of LMTK1 on Rab11A. In general, Rab small GTPases are regulated by a GEF, a GAP, and a GDP dissociation inhibitor. Each Rab is modulated by specific GEFs and GAPs, with some modulators influencing multiple Rabs, whereas only two GDP dissociation inhibitors regulate many Rabs (Cherfils and Zeghouf, 2013). Because the effect of LMTK1A was specific to Rab11 (Takano et al., 2010; Tsutsumi et al., 2010), we thought it likely that either LMTK1A inactivates a GEF or activates a GAP. SH3BP5 and Trapp2 complexes have recently been shown to be Rab11A GEFs (Sakaguchi et al., 2015; Thomas and Fromme, 2016), while Evi5, TBC1D9B, and TBC1D11 were reported as Rab11A GAPs (Dabbeekeh et al., 2007; Fuchs et al., 2007; Gallo et al., 2014). We tested the interaction of LMTK1A with these Rab11A GEFs and GAPs by coimmunoprecipitation. While none of the Rab11A GEFs bound to LMTK1A (data not shown), the GAPs, TBC1D9B and TBC1D11, bound to LMTK1A, with TBC1D9B displaying a stronger interaction (Fig. $8 C$; and data not shown). These results indicate that TBC1D9B is a LMTK1A binding protein, which may serve as a mediating factor between LMTK1 and Rab11A.
There has been only one paper describing the GAP activity of TBC1D9B on Rab11 (Gallo et al., 2014). Then, we tested whether TBC1D9B has Rab11A GAP activity in HEK293 cells via a pulldown assay using GST-Rabin8, an effector protein capable of binding to active Rab11A (Knödler et al., 2010; Homma and Fukuda, 2016). Rab11A bound to GST-Rabin8 was markedly reduced by cotransfection of TBC1D9B (Fig. 8D), confirming that TBC1D9B has Rab11A GAP activity in cultured cells. Next, we investigated the effect of LMTK1 on the interaction between Rab11A and TBC1D9B by coimmunoprecipitation. TBC1D9B bound to Rab11A, but the binding was not affected by the activity of LMTK1A (Fig. 8E). To determine whether LMTK1 regulates the GAP activity of TBC1D9B on Rab11A, we examined Rab11A activity after treatment with LMTK1A wt and kn constructs. Treatment of cells with LMTK1A wt significantly reduced the level of active Rab11A bound to Rabin8, compared with untreated cells or cells treated with LMTK1 kn (Fig. $8 F, G ; F_{(2,6)}=44$ for one-way ANOVA, $p=0.0002$ for wt vs kn, Tukey's multiple-comparisons test). These data indicate that LMTK1A regulates the GAP activity of TBC1D9B on Rab11A through its kinase activity.

\section{LMTK1A regulates spine formation through TBC1D9B}

TBC1D9B is a member of Tre2-Bub2-Cdc16 (TBC) domaincontaining proteins (Fig. 9A) (Fukuda, 2011). Currently, the biological function of TBC1D9B is unknown, and its expression in nervous tissue is unreported. Since there is no specific antibody 
A

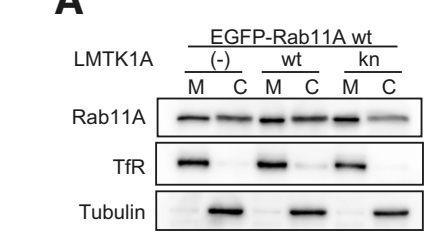

B
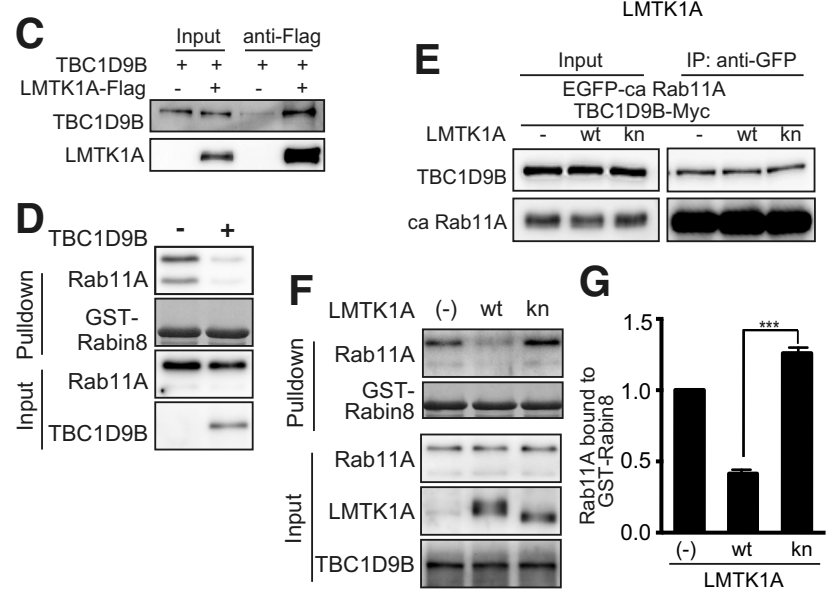

Figure 8. LMTK1A binds to TBC1D9B, a Rab11A GAP.A, Immunoblots of Rab11Ain the membrane (M) or cytosolic (C) fractions of Neuro-2a cells. Cell homogenates were fractionated into membrane and cytosolic fractions by centrifugation. The Ifreceptor (TFR) and tubulin (Tub) were used as markers of the membrane and cytosolic fractions, respectively. $\boldsymbol{B}$, The ratio of Rab11A in the membrane fraction is estimated and shown as mean \pm SEM $(n=3)$. C, The binding of LMTK1A with TBC1D9B. Neuro-2a cells were transfected with LMTK1A-Flag and EGFP-TBC1D9B. LMTK1-Flag was immunoprecipitated with anti-Flag, and TBC1D9B was detected with anti-GFP antibody. $\boldsymbol{D}$, The effect of TBC1D9B on the Rab11A activity. Rab11A was transfected in the presence $(+)$ or absence $(-)$ of TBC1D9B in HEK293 cells. Active Rab11A was pulled down with GST-Rabin8. Rab11A and TBC1D9B were detected by immunoblotting with anti-Rab11 and anti-Myc antibodies, respectively. $\boldsymbol{E}$, Neuro-2a cells were transfected with TBC1D9B-Myc, EGFP-ca Rab11A (070L) together with LMTK1AFlag wt or LMTK1A-Flag kn. Rab11A was immunoprecipitated with an anti-GFP antibody. TBC1D9B was detected with an anti-Myc antibody. $\boldsymbol{F}$, The effect of LMTK1 on the Rab11A GAP activity of TBC1D9B. HEK293 cells were transfected with EGFP-Rab11A, TBC1D9B, and LMTK1A wt or LMTK1A kn. The cell extract was incubated with GST-Rabin8 bound to GSH beads. Rab11A bound to GSTRabin8 was immunoblotted with anti-Rab11 antibody. LMTK1A, TBC1D9B, and Rabin8 were detected as described above. G, The ratio of active Rab11A bound to GST-Rabin8 is estimated and shown as mean \pm SEM $(n=3) .{ }^{* *} p<0.01,{ }^{* * *} p<0.001$.

against TBC1D9B, we determined whether TBC1D9B is expressed in mouse brain by RT-PCR. Using an adult mouse brain cDNA library, we detected a TBC1D9B transcript, validating its expression in nervous tissue (Fig. 9B). Next, we examined the role of TBC1D9B in neuronal spine formation by knockdown experiments. We prepared four shRNAs (\#1-\#4) against mouse TBC1D9B using HEK293 cells, and found that all four shTBC1D9B reduced the expression of cotransfected TBC1D9B by $>75 \%$ (Fig. 9C). We used shTBC1D9B \#2 and \#4 to reduce the expression of TBC1D9B in mouse primary neurons. Transfection of both TBC1D9B knockdown vectors increased spine density (Fig. $9 D, E ; F_{(2,31)}=20.1$ for one-way ANOVA, $p=0.0002$ for Scr vs \#2; $p<0.0001$ for Scr vs \#4, Tukey's multiple-comparisons test), indicating TBC1D9B plays a role in spine formation.

We confirmed the specificity of knockdown results by cotransfection of the knockdown vector-resistant human TBC1D9B (hTBC1D9B) (Fig. 9A). hTBC1D9B was expressed even in the presence of shTBC1D9B \#4, but not \#2, indicating its resistance (Fig. 9F). Cotransfection of hTBC1D9B reduced the spine density in neurons expressing the shTBC1D9B knockdown vector to the level of the control neurons (Fig. $9 G ; F_{(2,29)}=23.89$ for one- way ANOVA, $p<0.0001$ for Cont vs KD; $p<0.0001$ for Res vs $\mathrm{KD}$, Tukey's multiple-comparisons test). These results indicate the involvement of TBC1D9B in spine formation.

Next, we tested the effect of TBC1D9B overexpression on the spine density in primary neurons. As a control, we constructed an inactive form of TBC1D9B by mutating three residues in its TBC domain to alanine (Arg559, Tyr592, and Glu594: RYQ/AAA, 3A), as previously published (Gallo et al., 2014). TBC1D9B wt reduced spine density, as was anticipated, due to its intact GAP activity and regulation of Rab11A. More importantly, expression of the RYQ/AAA mutant increased spine density in hippocampal neurons. This may be a dominant-negative effect of suppressing endogenous TBC1D9B (Fig. 9H,I; $F_{(2,48)}=21.5$ for one-way ANOVA, $p=0.0041$ for $(-)$ vs wt; $p=0.0046$ for $(-)$ vs $3 \mathrm{~A} ; p<$ 0.0001 for wt vs $3 \mathrm{~A}$, Tukey's multiple-comparisons test).

Finally, we examined the effect of TBC1D9B on spine formation in LMTK1-knockdown neurons to determine their relationship. TBC1D9B wt expression negated the effect of LMTK1 knockdown and further reduced neuronal spine density (Fig. 9J, K; $p=0.0128$ for two-way ANOVA; $p=0.021$ for miR-Scr(-) vs miR-Scr-TBC1D9B wt, $p=0.9903$ for miR-Scr-TBC1D9B wt vs miR-LMTK1-TBC1D9B wt, Tukey's multiple-comparisons test). The dominant-negative mutant (RYQ/AAA) of TBC1D9B did not increase the spine density more than the LMTK1 knockdown (Fig. 9J,K). These results clearly indicate that TBC1D9B functions downstream of LMTK1 ( $p>0.9999$ for miR-Scr-TBC1D9B 3A vs miR-LMTK1-TBC1D9B 3A, Tukey's multiple-comparisons test).

\section{Discussion}

In this study, we show, for the first time, that dendritic spine formation is regulated by LMTK1A, a Ser/Thr kinase bound to Rab11-positive recycling endosomes. LMTK1 prevents excessive spine formation by suppressing Rab11A activity. We further find that TBC1D9B, a Rab11A GAP, plays a role in spine formation by mediating the influence of LMTK1 on Rab11A. We propose that LMTK1-TBC1D9B-Rab11 is a novel signaling cascade in endosomal transport, which regulates dendritic spine formation, maturation and density in neurons.

Dendritic spines are postsynaptic protrusions on the dendrites of neurons, which receive excitatory signals from presynaptic neurons. Its formation, including spine size and number, are crucial for proper neuronal circuit wiring and brain function (Yuste, 2011). Previous studies have identified many proteins involved in spine formation, but the molecular mechanism regulating delivery of membrane components to spines remains unknown. LMTK1 is a novel modulator of spine formation, whose depletion increases the density of spines (Figs. 1, 2).

Spines are dynamic structures that can rapidly change in size and shape throughout development and in response to external stimuli. Using real-time imaging, we demonstrate that loss of LMTK1 increases the formation of spines (Fig. 5). Considering that LMTK1 is a negative regulator of endosomal trafficking, downregulation of LMTK1 may stimulate delivery of membrane proteins, such as neurotransmitter receptors and synaptic adhesion molecules to spines, resulting in a surplus of membrane components and overformation of spines. In contrast, under basal conditions, LMTK1 prevents aberrant or excessive spine development by limiting the supply of membrane components.

Vesicular transport is the primary mode of lipid and protein delivery to spines, and Rab proteins strongly influence this trafficking (Fukuda, 2008; Stenmark, 2009; Hutagalung and Novick, 2011). Recently, several studies have indicated the involvement of Rab11-associated vesicles in this process (Park et al., 2006; Hoogen- 
raad et al., 2010; Bodrikov et al., 2017). Rab11A is a classical Rab, whose role in trafficking recycling endosomes has been well documented. In contrast, the regulation of Rab11A activity in neurons is poorly understood. LMTK1 is one of only a few known upstream regulators of Rab11. LMTK1 is transported in axons and dendrites on Rab11-positive endosomes, and previous studies demonstrate that LMTK1 knockdown increases anterograde transport and the total distance traveled of Rab11-positive endosomes in axons and dendrites (Takano et al., 2014). By combining this knowledge with constitutively active or inactive Rab11 mutants, we are able to dissect the relationship between LMTK1 and Rab11 in neuronal spine formation. We show that the effect of LMTK1 knockdown is abrogated by the expression of a constitutively active Rab11A. These results clearly indicate that Rab11A activity is regulated by LMTK1A (Takano et al., 2012). However, the regulation is indirect, and how LMTK1 transmits its activity to Rab11 has remained an open question.

LMTK1 is a unique member of protein kinases with complex kinase activity and regulatory mechanisms. LMTK1 was initially suggested to be a Tyr kinase from the homology of its kinase domain sequence to that of receptor tyrosine kinases (Gaozza et al., 1997). But one of its family members, LMTK2, previously called KPI-1 or BREK, was later reported to be a Ser/Thr kinase (Kawa et al., 2004; Wang and Brautigan, 2006). As a member of the LMTK kinase family, LMTK1 is now considered a Ser/Thr kinase; however, its kinase activity has not yet been distinctly demonstrated, and its in vitro and in vivo substrates remain unidentified. Asp206 in the kinase domain of LMTK1A corresponds to the amino acid critical for kinase activity of many other protein kinases. Because a LMTK1A D206V mutant exhibits phenotypes similar to those of LMTK1 knockdowns, it appears that LMTK1 functions as a protein kinase. In addition, our spine formation analyses support this kinase role.

LMTK1A is subjected to post-translational modification, such as phosphorylation by $\mathrm{Cdk} 5 / \mathrm{p} 35$ or palmitoylation (Tsutsumi et al., 2008, 2010; Manser et al., 2012). An Ala mutation at the Ser34 Cdk5 phosphorylation site has been shown to display similar phenotypes to LMTK1 kn, indicating that LMTK1 activity is controlled by $\mathrm{Cdk} 5 / \mathrm{p} 35$ phosphorylation. Cdk5/p35 is active in resting neurons (Kimura et al., 2014) and can bind recycling endosomes through p35 N-terminal myristoylation (Asada et al., 2008). Together, these results suggest that LMTK1 is activated on endosomes by Cdk5/p35 (Fig. 10).
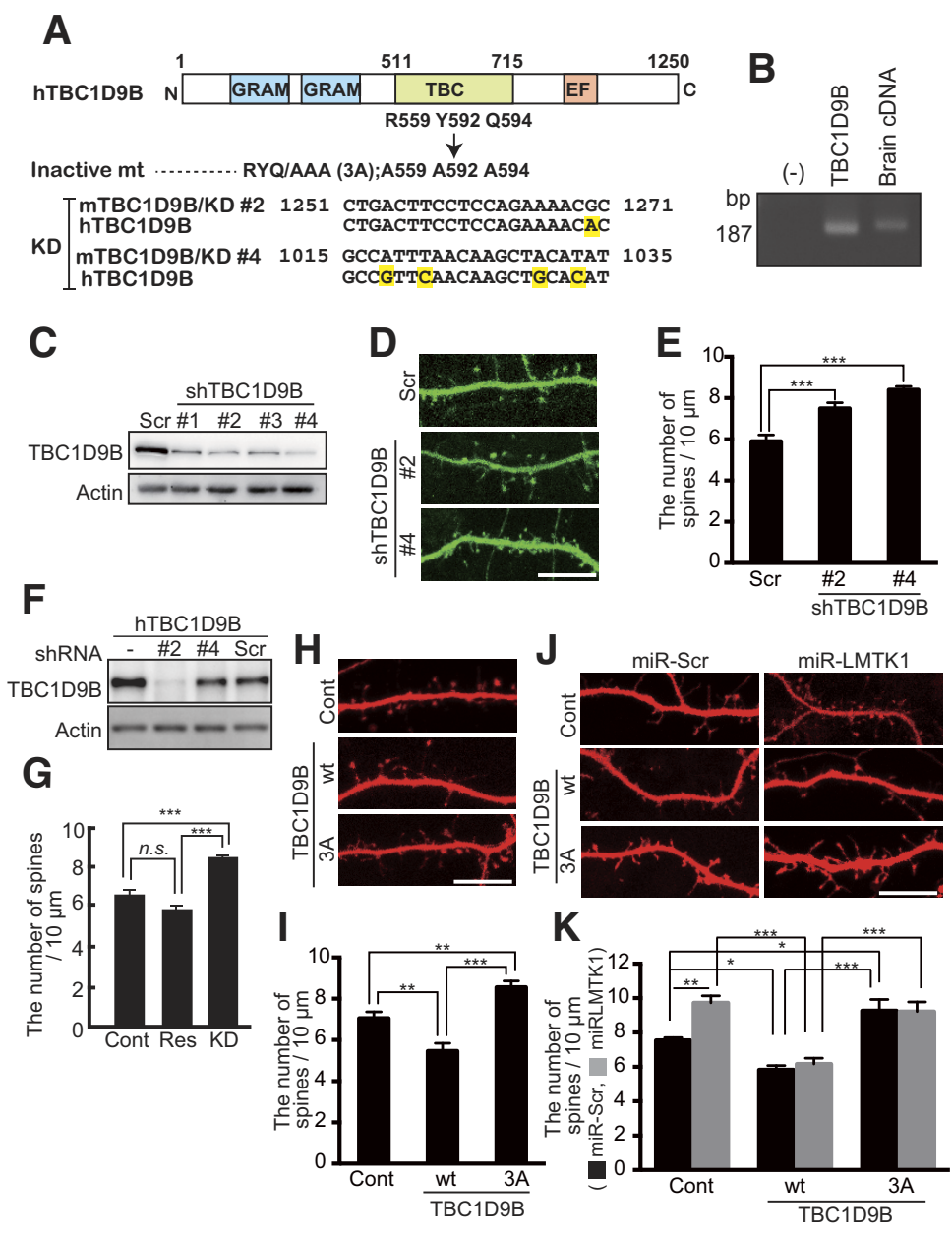

Figure 9. LMTK1 regulates spine formation through TBC1D9B. $A$, The domain structure of human TBC1D9B (hTBC1D9B). TBC1D9B is a TBC domain containing protein with Rab11 GAP activity (Fukuda, 2011). The inactive mutant (mt) of TBC1B9D was constructed by mutating Arg559, Tyr592, and Gln594 in the TBC domain to Ala (RYQ/AAA, 3A) (Gallo et al., 2014). DNA sequences of mouse TBC1D9B ( $m$ TBC1D9B) knockdown vectors (shRNA \#2 and shRNA \#4) and their corresponding regions in human TBC1D9B ( $\mathrm{CTBC1D9B)}$. The DNA sequence of the shRNA is presented below $\mathrm{mTBC} 1 \mathrm{D} 9 \mathrm{~B} \# 2$ and \#4, with base pair differences highlighted in yellow. $\boldsymbol{B}$, Agarose gel electrophoresis of PCR products using primers specific for TBC1D9B (813-999 bp) and a CDNA library of mouse brain as a template. ( - ), no template; mouse TBC1D9B-Myc was used as a positive control. $C$, Effect of knockdown vectors on TBC1D9B expression in HEK293 cells. HEK293 cells were transfected with mouse TBC1D9B-Myc and shTBC1D9B knockdown vectors $1-4$. The expression of TBC1D9B was detected by immunoblotting with an anti-Myc antibody. Actin is the loading control. $D$, Effect of TBC1D9B knockdown on spine density of primary neurons. Hippocampal neurons were transfected with GFP and sh-Scr or sh-TBC1D9B \#2 or \#4 at $10 \mathrm{DIV}$, and dendritic spines were observed at $15 \mathrm{DIV}$. Scale bar, $10 \mu \mathrm{m}$. $\boldsymbol{E}$, The density of spines is measured and shown as mean $\pm \operatorname{SEM}(n=10$ for $S c r ; n=14$ for \#2;n=10 for \#4).F, Expression of hTBC1D9B in the presence of shRNA \#2 or \#4 in HEK293 cells. G, Cotransfection of hippocampal neurons with shRNA \#4 and hTBC1D9B (Res) reverted the number of dendritic spines to control levels (Cont) ( $n=12$ for Cont, $n=10$ for Res, and $n=10$ for KD). ${ }^{* * *} p<0.001$. $F_{(2,29)}=23.89$ for one-way ANOVA, $p<0.001$ for Cont vs KD, $p<0.0001$ for Res vs KD (Turkey's multiple-comparisons test). $\boldsymbol{H}$, Hippocampal neurons were transfected with TBC1D9B wt, its inactive mutant RYQ/AAA (3A), or DsRed and an empty vector ( - ) at $10 \mathrm{DIV}$. Dendritic spines were observed at $15 \mathrm{DIV}$. Scale bar, $10 \mu \mathrm{m}$. $I$, The density of spines is measured and expressed as mean \pm SEM ( $n=18$ for control; $n=15$ for TBC1D9B wt; $n=18$ for 3 A mutant). J, Hippocampal neurons at 10 DIV were transfected with DsRed and miR-Scr or miR-LMTK1 together with either of TBC1D9B wt, its inactive mutant 3A, or an empty vector $(-)$, and dendritic spines were observed at 15 DIV. Scale bar, $10 \mu \mathrm{m}$. $K$, The density of spines is indicated as mean $\pm \operatorname{SEM}(n=22, n=12$, and $n=11$ for miR-Scr and empty vector, TBC1D9B wt, or 3A mutant; $n=11, n=18$, and $n=14$ for miR-LMTK1 and empty vector, TBC1D9B wt, or RYQ/AAA mutant. ${ }^{*} p<0.05,{ }^{* *} p<0.01$.

Rab small GTPases are regulated by GEFs and GAPs. Several Rab11A-specific GEFs and GAPs have recently been identified, but their precise regulatory mechanisms were not fully elucidated. In this study, we find that TBC1D9B, a Rab11A GAP, regulates spine formation. The GAP activity of TBC1D9B on Rab11A has been studied in vitro and in MDCK cells (Gallo et al., 2014), but its function in other cells and tissues has not been 
A

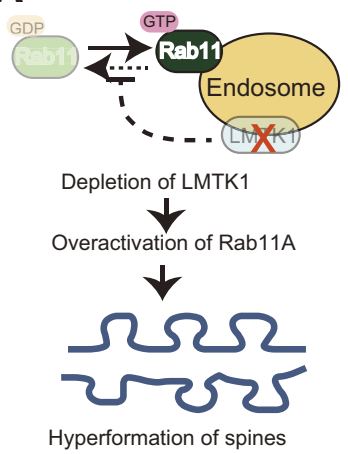

B

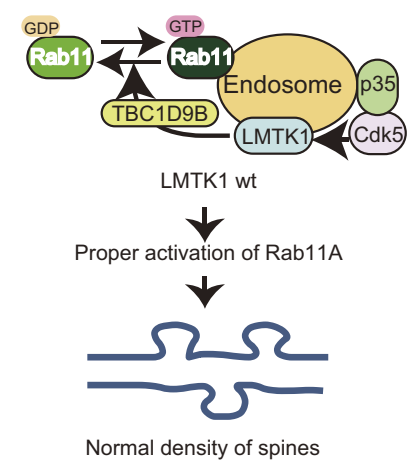

Figure 10. The regulation of the Rab11A activity on endosomes and its role in spine formation. $A$, Downregulation of LMTK1 on endosomes results in overactivation of Rab11A, leading to excessive dendritic spine formation. $\boldsymbol{B}$, The proper regulation of Rab11A is important for controlling appropriate spine number. The Cdk5-LMTK1A-TBC1D9B pathway is the upstream cascade of Rab11A endosomal regulation. Cdk5/p35regulates LMTK1A activity by phosphorylation at Ser34. LMTK1 activates TBC1D9B, leading to inactivation of Rab11A activity. Interestingly, Cdk5/p35, LMTK1, and Rab11A bind to endosomes through lipid modification, myristoylation, palmitoylation, and farnesylation. Therefore, the regulation cascade operates on the endosome.

addressed. Here, overexpression of TBC1D9B reduces spine density (Fig. 9H,I), and its knockdown increases spine density in primary neurons (Fig. 9D,E). These effects of TBC1D9B on spine formation are mediated by Rab11A GAP activity (Fig. $9 \mathrm{~J}, K$ ). This is the first evidence that TBC1D9B works as a Rab11A GAP in a particular cellular activity. More importantly, TBC1D9B functions downstream of LMTK1. Thus, the LMTK1-TBC1D9B-Rab11A cascade is a novel signaling pathway regulating endosomal trafficking for spine and synapse formation (Fig. 10).

Rab11-positive endosomes are transported along microtubules longitudinally in the dendritic shaft. These vesicles must terminate their longitudinal movement and travel laterally to the surface of dendrites at precise locations where spines have formed, or will form. Rab11-positve endosomes are found in spines (Gerges et al., 2006; Park et al., 2006; Brown et al., 2007), and Esteves da Silva et al. (2015) have directly observed movement of Rab11-positive endosomes into spines by real-time imaging. Adding to these findings, we observe LMTK1A bound to Rab11A-positive vesicles in spines (Fig. 7C), suggesting that LMTK1A is also transported to spines on Rab11-positive endosomes.

While a recent report indicates that dendritic spines may contain microtubules (Schätzle et al., 2018), they are generally thought to be actin-rich structures. Therefore, Rab11-positive vesicles may have to alter their motor protein and cytoskeletal track to enter dendritic spines. As evidence for this hypothesis, LMTK2 is known to be a myosin VI-binding protein (Chibalina et al., 2007; Inoue et al., 2008) and myosin VI is shown to regulate the spine number and structure (Osterweil et al., 2005). Furthermore, LMTK2 and LMTK1 both bind Cdk5/p35 at regions that overlap with LMTK2's myosin VI binding site, suggesting that LMTK1 may also bind myosin VI (Kesavapany et al., 2003). Thus, LMTK1 may also play a role in enabling the migration of Rabllpositive endosomes into spines.

In general, Rab11 conveys recycling endosomes to the cell periphery but does not control the final interaction with the plasma membrane. LMTK1 activation of TBC1D9B leads to inactivation of Rab11. Then, another protein must modulate endosomal movement into postsynaptic spines and their delivery to the membrane. Rab8 is one such possible candidate that has been shown to take over vesicle movement during primary cilia for- mation (Knödler et al., 2010). Vesicle exchange from Rab11 to Rab8 has also been reported with transport of AMPA receptors to dendritic spines (Brown et al., 2007). Additionally, we recently reported that endosomal vesicles are transferred from Rab11 to Rab8 at the growth cone of elongating axons (Furusawa et al., 2017). This handoff is mediated by GRAB, an effector of Rab11A and a GEF of Rab8. Interestingly, GRAB activity is regulated by Cdk5/p35 (Furusawa et al., 2017). Therefore, Cdk5/p35 inactivates Rab11 through the LMTK1-TBC1D9B cascade and, at the same time, activates Rab8 through GRAB to promote trafficking of endosomal vesicles into spines.

Spine density and maturation have been closely tied to higher neuronal function. Morphological studies of disease models and human brain samples suggest that a delicate balance exists between proper synapse formation and function. For instance, increased spine density has been linked to neurodevelopmental disorders, such as autism spectrum disorder (Hutsler and Zhang, 2010; Penzes et al., 2011). In particular, the autism-linked mutation of UBE3A is associated with increased spine density (Yi et al., 2015); and knockdown of TAOK2, a 16p11.2 locus associated with autism spectrum disorder, increases the number of immature spines (Yadav et al., 2017). Here, we demonstrate that downregulation of LMTK1 activity increases spine density. In addition, previous work illustrates that knockdown of LMTK1 induces excessive dendritic arborization during early postnatal development (Takano et al., 2014). Together, these data support a possible role of LMTK1 in neurodevelopment, and suggest that its dysfunction may contribute to disorders, such as autism spectrum disorder. Future studies investigating the functional and behavioral effects of LMTK1 in vivo may reveal how endosomal trafficking influences neurocognition and behavior.

\section{References}

Asada A, Yamamoto N, Gohda M, Saito T, Hayashi N, Hisanaga S (2008) Myristoylation of p39 and p35 is a determinant of cytoplasmic or nuclear localization of active cyclin-dependent kinase 5 complexes. J Neurochem 106:1325-1336.

Baker SJ, Sumerson R, Reddy CD, Berrebi AS, Flynn DC, Reddy EP (2001) Characterization of an alternatively spliced AATYK mRNA: expression pattern of AATYK in the brain and neuronal cells. Oncogene 20:1015-1021.

Bodrikov V, Pauschert A, Kochlamazashvili G, Stuermer CAO (2017) Reggie-1 and reggie-2 (flotillins) participate in Rablla-dependent cargo trafficking, spine synapse formation and LTP-related AMPA receptor (GluA1) surface exposure in mouse hippocampal neurons. Exp Neurol 289:31-45.

Bosch M, Hayashi Y (2012) Structural plasticity of dendritic spines. Curr Opin Neurobiol 22:383-388.

Brown TC, Correia SS, Petrok CN, Esteban JA (2007) Functional compartmentalization of endosomal trafficking for the synaptic delivery of AMPA receptors during long-term potentiation. J Neurosci 27:13311-13315.

Calabrese B, Wilson MS, Halpain S (2006) Development and regulation of dendritic spine synapses. Physiol 21:38-47.

Cherfils J, Zeghouf M (2013) Regulation of small GTPases by GEFs, GAPs, and GDIs. Physiol Rev 93:269-309.

Chibalina MV, Seaman MN, Miller CC, Kendrick-Jones J, Buss F (2007) Myosin VI and its interacting protein LMTK2 regulate tubule formation and transport to the endocytic recycling compartment. J Cell Sci 120: $4278-4288$.

Choquet D, Triller A (2013) The dynamic synapse. Neuron 80:691-703.

Dabbeekeh JT, Faitar SL, Dufresne CP, Cowell JK (2007) The EVI5 TBC domain provides the GTPase-activating protein motif for RAB11. Oncogene 26:2804-2808.

Dunaevsky A, Tashiro A, Majewska A, Mason C, Yuste R (1999) Developmental regulation of spine motility in the mammalian central nervous system. Proc Natl Acad Sci U S A 96:13438-13443.

Esteves da Silva M, Adrian M, Schätzle P, Lipka J, Watanabe T, Cho S, Futai K, Wierenga CJ, Kapitein LC, Hoogenraad CC (2015) Positioning of AMPA receptor-containing endosomes regulates synapse architecture. Cell Rep 13:933-943. 
Fuchs E, Haas AK, Spooner RA, Yoshimura S, Lord JM, Barr FA (2007) Specific Rab GTPase-activating proteins define the Shiga toxin and epidermal growth factor uptake pathways. J Cell Biol 177:1133-1143.

Fukuda M (2008) Regulation of secretory vesicle traffic by Rab small GTPases. Cell Mol Life Sci 65:2801-2813.

Fukuda M (2011) TBC proteins: GAPs for mammalian small GTPase Rab? Biosci Rep 31:159-168.

Furusawa K, Asada A, Urrutia P, Gonzalez-Billault C, Fukuda M, Hisanaga SI (2017) Cdk5 regulation of the GRAB-mediated Rab8-Rab11 cascade in axon outgrowth. J Neurosci 37:790-806.

Gagnon KB, England R, Diehl L, Delpire E (2007) Apoptosis-associated tyrosine kinase scaffolding of protein phosphatase 1 and SPAK reveals a novel pathway for na-K-2C1 cotransporter regulation. Am J Physiol Cell Physiol 292:C1809-C1815.

Gallo LI, Liao Y, Ruiz WG, Clayton DR, Li M, Liu YJ, Jiang Y, Fukuda M, Apodaca G, Yin XM (2014) TBC1D9B functions as a GTPase-activating protein for Rab1la in polarized MDCK cells. Mol Biol Cell 25:3779-3797.

Gaozza E, Baker SJ, Vora RK, Reddy EP (1997) AATYK: a novel tyrosine kinase induced during growth arrest and apoptosis of myeloid cells. Oncogene 15:3127-3135.

Gerges NZ, Backos DS, Rupasinghe CN, Spaller MR, Esteban JA (2006) Dual role of the exocyst in AMPA receptor targeting and insertion into the postsynaptic membrane. EMBO J 25:1623-1634.

Homma Y, Fukuda M (2016) Rabin8 regulates neurite outgrowth in both GEF activity-dependent and -independent manners. Mol Biol Cell 27:2107-2118.

Honma N, Asada A, Takeshita S, Enomoto M, Yamakawa E, Tsutsumi K, Saito T, Satoh T, Itoh H, Kaziro Y, Kishimoto T, Hisanaga S (2003) Apoptosis-associated tyrosine kinase is a Cdk5 activator $\mathrm{p} 35$ binding protein. Biochem Biophys Res Commun 310:398-404.

Hoogenraad CC, Popa I, Futai K, Martinez-Sanchez E, Wulf PS, van Vlijmen T, Dortland BR, Oorschot V, Govers R, Monti M, Heck AJ, Sheng M, Klumperman J, Rehmann H, Jaarsma D, Kapitein LC, van der Sluijs P (2010) Neuron specific Rab4 effector GRASP-1 coordinates membrane specialization and maturation of recycling endosomes. PLoS Biol 8:e1000283.

Hutagalung AH, Novick PJ (2011) Role of Rab GTPases in membrane traffic and cell physiology. Physiol Rev 91:119-149.

Hutsler JJ, Zhang H (2010) Increased dendritic spine densities on cortical projection neurons in autism spectrum disorders. Brain Res 1309:83-94.

Inoue T, Kon T, Ohkura R, Yamakawa H, Ohara O, Yokota J, Sutoh K (2008) BREK/LMTK2 is a myosin VI-binding protein involved in endosomal membrane trafficking. Genes Cells 13:483-495.

Itoh T, Kanno E, Uemura T, Waguri S, Fukuda M (2011) OATL1, a novel autophagosome-resident Rab33B-GAP, regulates autophagosomal maturation. J Cell Biol 192:839-853.

Kawa S, Fujimoto J, Tezuka T, Nakazawa T, Yamamoto T (2004) Involvement of BREK, a serine/threonine kinase enriched in brain, in NGF signalling. Genes Cells 9:219-232.

Kawauchi T, Chihama K, Nishimura YV, Nabeshima Y, Hoshino M (2005) MAP1B phosphorylation is differentially regulated by Cdk5/p35, Cdk5/ p25, and JNK. Biochem Biophys Res Commun 331:50-55.

Kesavapany S, Lau KF, Ackerley S, Banner SJ, Shemilt SJ, Cooper JD, Leigh PN, Shaw CE, McLoughlin DM, Miller CC (2003) Identification of a novel, membrane-associated neuronal kinase, cyclin-dependent kinase 5/p35-regulated kinase. J Neurosci 23:4975-4983.

Kimura T, Ishiguro K, Hisanaga S (2014) Physiological and pathological phosphorylation of tau by Cdk5. Front Mol Neurosci 7:65.

Knödler A, Feng S, Zhang J, Zhang X, Das A, Peränen J, Guo W (2010) Coordination of Rab8 and Rab11 in primary ciliogenesis. Proc Natl Acad Sci U S A 107:6346-6351.

Lei W, Omotade OF, Myers KR, Zheng JQ (2016) Actin cytoskeleton in dendritic spine development and plasticity. Curr Opin Neurobiol 39:86-92.

Manser C, Vagnoni A, Guillot F, Davies J, Miller CC (2012) Cdk5/p35 phosphorylates lemur tyrosine kinase-2 to regulate protein phosphatase-1C phosphorylation and activity. J Neurochem 121:343-348.

Maxfield FR, McGraw TE (2004) Endocytic recycling. Nat Rev Mol Cell Biol $5: 121-132$.

Osterweil E, Wells DG, Mooseker MS (2005) A role for myosin VI in postsynaptic structure and glutamate receptor endocytosis. J Cell Biol 168:329-338.

Park M, Salgado JM, Ostroff L, Helton TD, Robinson CG, Harris KM, Ehlers
MD (2006) Plasticity-induced growth of dendritic spines by exocytic trafficking from recycling endosomes. Neuron 52:817-830.

Penzes P, Cahill ME, Jones KA, VanLeeuwen JE, Woolfrey KM (2011) Dendritic spine pathology in neuropsychiatric disorders. Nat Neurosci 14:285-293

Riedl J, Crevenna AH, Kessenbrock K, Yu JH, Neukirchen D, Bista M, Bradke F, Jenne D, Holak TA, Werb Z, Sixt M, Wedlich-Soldner R (2008) Lifeact: a versatile marker to visualize F-actin. Nat Methods 5:605-607.

Sakaguchi A, Sato M, Sato K, Gengyo-Ando K, Yorimitsu T, Nakai J, Hara T, Sato K, Sato K (2015) REI-1 is a guanine nucleotide exchange factor regulating RAB-11 localization and function in C. elegans embryos. Dev Cell 35:211-221.

Schätzle P, Esteves da Silva M, Tas RP, Katrukha EA, Hu HY, Wierenga CJ, Kapitein LC, Hoogenraad CC (2018) Activity-dependent actin remodeling at the base of dendritic spines promotes microtubule entry. Curr Biol 28:2081-2093.e6.

Sharma G, Tsutsumi K, Saito T, Asada A, Ando K, Tomomura M, Hisanaga SI (2016) Kinase activity of endosomal kinase LMTK1A regulates its cellular localization and interactions with cytoskeletons. Genes Cells 21:1080-1094.

Spires-Jones TL, Hyman BT (2014) The intersection of amyloid beta and tau at synapses in Alzheimer's disease. Neuron 82:756-771.

Stenmark H (2009) Rab GTPases as coordinators of vesicle traffic. Nat Rev Mol Cell Biol 10:513-525.

Tabata H, Nakajima K (2001) Efficient in utero gene transfer system to the developing mouse brain using electroporation: visualization of neuronal migration in the developing cortex. Neurosci 103:865-872.

Takano T, Tsutsumi K, Saito T, Asada A, Tomomura M, Fukuda M, Hisanaga S (2010) AATYK1A phosphorylation by Cdk5 regulates the recycling endosome pathway. Genes Cells 15:783-797.

Takano T, Tomomura M, Yoshioka N, Tsutsumi K, Terasawa Y, Saito T, Kawano H, Kamiguchi H, Fukuda M, Hisanaga S (2012) LMTK1/ AATYK1 is a novel regulator of axonal outgrowth that acts via Rab11 in a Cdk5-dependent manner. J Neurosci 32:6587-6599.

Takano T, Urushibara T, Yoshioka N, Saito T, Fukuda M, Tomomura M, Hisanaga S (2014) LMTK1 regulates dendritic formation by regulating movement of Rab11A-positive endosomes. Mol Biol Cell 25:1755-1768.

Thomas LL, Fromme JC (2016) GTPase cross talk regulates TRAPPII activation of Rab11 homologues during vesicle biogenesis. J Cell Biol 215:499-513

Tomomura M, Fernandez-Gonzales A, Yano R, Yuzaki M (2001) Characterization of the apoptosis-associated tyrosine kinase (AATYK) expressed in the CNS. Oncogene 20:1022-1032.

Tomomura M, Morita N, Yoshikawa F, Konishi A, Akiyama H, Furuichi T, Kamiguchi H (2007) Structural and functional analysis of the apoptosisassociated tyrosine kinase (AATYK) family. Neuroscience 148:510-521.

Tsuboi T, Fukuda M (2006) Rab3A and Rab27A cooperatively regulate the docking step of dense-core vesicle exocytosis in PC12 cells. J Cell Sci 119:2196-2203

Tsutsumi K, Tomomura M, Furuichi T, Hisanaga S (2008) Palmitoylationdependent endosomal localization of AATYK1A and its interaction with Src. Genes Cells 13:949-964.

Tsutsumi K, Takano T, Endo R, Fukuda M, Ohshima T, Tomomura M, Hisanaga S (2010) Phosphorylation of AATYK1 by Cdk5 suppresses its tyrosine phosphorylation. PLoS One 5:e10260.

Ullrich O, Reinsch S, Urbé S, Zerial M, Parton RG (1996) Rab11 regulates recycling through the pericentriolar recycling endosome. J Cell Biol 135:913-924.

Ultanir SK, Hertz NT, Li G, Ge WP, Burlingame AL, Pleasure SJ, Shokat KM, Jan LY, Jan YN (2012) Chemical genetic identification of NDR1/2 kinase substrates AAK1 and Rabin8 uncovers their roles in dendrite arborization and spine development. Neuron 73:1127-1142.

Wang H, Brautigan DL (2006) Peptide microarray analysis of substrate specificity of the transmembrane Ser/Thr kinase KPI-2 reveals reactivity with cystic fibrosis transmembrane conductance regulator and phosphorylase. Mol Cell Proteom 5:2124-2130.

Yadav S, Oses-Prieto JA, Peters CJ, Zhou J, Pleasure SJ, Burlingame AL, Jan LY, Jan YN (2017) TAOK2 kinase mediates PSD95 stability and dendritic spine maturation through Septin7 phosphorylation. Neuron 93:379-393.

Yi JJ, Berrios J, Newbern JM, Snider WD, Philpot BD, Hahn KM, Zylka M] (2015) An autism-linked mutation disables phosphorylation control of UBE3A. Cell 162:795-807.

Yuste R (2011) Dendritic spines and distributed circuits. Neuron 71:772-781. 